\title{
New constraints on the planetary system around the young active star AU Mic
}

\section{Two transiting warm Neptunes near mean-motion resonance}

\author{
E. Martioli ${ }^{1,2}$, G. Hébrard ${ }^{1,3}$, A. C. M. Correia ${ }^{4,5}$, J. Laskar ${ }^{5}$, and A. Lecavelier des Etangs ${ }^{1}$ \\ ${ }^{1}$ Institut d'Astrophysique de Paris, CNRS, UMR 7095, Sorbonne Université, 98 bis bd Arago, 75014 Paris, France \\ e-mail: martioli@iap.fr \\ ${ }^{2}$ Laboratório Nacional de Astrofísica, Rua Estados Unidos 154, 37504-364, Itajubá - MG, Brazil \\ ${ }^{3}$ Observatoire de Haute Provence, St Michel l'Observatoire, France \\ ${ }^{4}$ CFisUC, Department of Physics, University of Coimbra, 3004-516 Coimbra, Portugal \\ 5 IMCCE, UMR8028 CNRS, Observatoire de Paris, PSL University, Sorbonne Univ., 77 av. Denfert-Rochereau, 75014 Paris, \\ France
}

Received 24 December 2020 / Accepted 27 March 2021

\begin{abstract}
AU Microscopii (AU Mic) is a young, active star whose transiting planet was recently detected. Here, we report our analysis of its TESS light curve, where we modeled the BY Draconis type quasi-periodic rotational modulation by starspots simultaneously to the flaring activity and planetary transits. We measured a flare occurrence rate in AU Mic of 6.35 flares per day for flares with amplitudes in the range of $0.06 \%<f_{\max }<1.5 \%$ of the star flux. We employed a Bayesian Markov chain Monte Carlo analysis to model the five transits of AU Mic b observed by TESS, improving the constraints on the planetary parameters. The measured planet-to-star effective radius ratio of $R_{\mathrm{p}} / R_{\star}=0.0496 \pm 0.0007$ implies a physical radius of $4.07 \pm 0.17 R_{\oplus}$ and a planet density of $1.4 \pm 0.4 \mathrm{~g} \mathrm{~cm}^{-3}$, confirming that AU Mic b is a Neptune-size moderately inflated planet. While a single feature possibly due to a second planet was previously reported in the former TESS data, we report the detection of two additional transit-like events in the new TESS observations of July 2020. This represents substantial evidence for a second planet (AU Mic c) in the system. We analyzed its three available transits and obtained an orbital period of $18.859019 \pm 0.000016 \mathrm{~d}$ and a planetary radius of $3.24 \pm 0.16 R_{\oplus}$, which defines AU Mic c as a warm Neptune-size planet with an expected mass in the range of $2.2 M_{\oplus}<M_{\mathrm{c}}<25.0 M_{\oplus}$, estimated from the population of exoplanets of similar sizes. The two planets in the AU Mic system are in near 9:4 mean-motion resonance. We show that this configuration is dynamically stable and should produce transit-timing variations (TTV). Our non-detection of significant TTV in AU Mic b suggests an upper limit for the mass of AU Mic c of $<7 M_{\oplus}$, indicating that this planet is also likely to be inflated. As a young multi-planet system with at least two transiting planets, AU Mic becomes a key system for the study of atmospheres of infant planets and of planet-planet and planet-disk dynamics at the early stages of planetary evolution.
\end{abstract}

Key words. planetary systems - stars: individual: AU Mic - stars: activity - techniques: photometric

\section{Introduction}

Young planetary systems represent an opportunity to observe planets in the early stages of planetary formation when gravitational interactions have not significantly changed the initial configuration of the system. AU Microscopii (AU Mic) is a particularly interesting young system, with an estimated age of $22 \pm 3$ Myr (Mamajek \& Bell 2014), which is located at a distance of only $9.7248 \pm 0.0046$ pc (Gaia Collaboration 2018). Table 1 summarizes the star parameters of AU Mic that are relevant for this work. The AU Mic host is an M1 star with a spatially resolved edge-on debris disk (Kalas et al. 2004) and at least one transiting planet, AU Mic b (Plavchan et al. 2020). This Neptune-size planet is in a 8.5-d prograde orbit aligned with the stellar rotation axis (Martioli et al. 2020; Hirano et al. 2020; Palle et al. 2020). While Plavchan et al. (2020) only reported an upper limit on the mass of AU Mic b, Klein et al. (2021) measured $17.1_{-4.5}^{+4.7} M_{\oplus}$ thanks to infrared observations secured with the SPIRou spectropolarimeter (Donati et al. 2020). Plavchan et al. (2020) has also reported the detection of an isolated transit event of a second possible candidate planet, hereafter referred to as AU Mic c.

The notion that AU Mic could be a host for several planets is not a surprising one. Indeed, the occurrence rate of planets with a radius between $0.5 R_{\oplus}$ and $4.0 R_{\oplus}$ and a period between 0.5 and $256 \mathrm{~d}$ is estimated as $8.4_{-1.1}^{+1.2}$ planets per M dwarf (Hsu et al. 2020), although the occurrence rate of more massive planets in $\mathrm{M}$ dwarfs decreases significantly with mass (e.g., Bonfils et al. 2013). Moreover, provided the fact that AU Mic has an edgeon debris disk and that AU Mic b is a transiting planet with an aligned orbit, the chances that other planets also reside in coplanar orbits increase and, therefore, possible close-in additional planets are also likely to transit the star.

AU Mic is a magnetically active star with strong flaring activity (Robinson et al. 2001). Its surface is largely filled by starspots, producing a BY Draconis-type light curve with a quasi-periodic rotational modulation and a period of $4.863 \pm 0.010 \mathrm{~d}$ (Plavchan et al. 2020). As in other active stars, the strong flaring and magnetic activity of AU Mic makes it more difficult to detect planetary transits in a photometric time series, especially for 
Table 1. AU Mic star parameters.

\begin{tabular}{lcc}
\hline \hline Parameter & Value & Ref. \\
\hline Effective temperature & $3700 \pm 100 \mathrm{~K}$ & 1 \\
Star mass & $0.50 \pm 0.03 M_{\odot}$ & 1 \\
Star radius & $0.75 \pm 0.03 R_{\odot}$ & 2 \\
Rotation period & $4.863 \pm 0.010 \mathrm{~d}$ & 1 \\
Age & $22 \pm 3 \mathrm{Myr}$ & 3 \\
Distance & $9.7248 \pm 0.0046$ parsec & 4 \\
Linear limb dark. coef. & 0.2348 & 5 \\
Quadratic limb dark. coef. & 0.3750 & 5 \\
\hline
\end{tabular}

References. (1) Plavchan et al. (2020); (2) White et al. (2015); (3) Mamajek \& Bell (2014); (4) Gaia Collaboration (2018); (5) Claret (2018).

small planets where the occurrence rate is higher. In this work, we present an analysis of the Transiting Exoplanet Survey Satellite (TESS) data of AU Mic, including the new observations obtained in July 2020, where we implement a multi-flare model combined with the starspots model, improving the constraints on the planetary parameters from transit modeling and allowing for the detection of two additional transits of the second candidate planet AU Mic c.

\section{TESS light curve}

AU Mic was observed by TESS (Ricker et al. 2015) in Sector 1 from 2018-Jul.-25 to 2018-Aug.-22, in cycle 1, camera 1. Then it was observed again in Sector 27 from 2020-Jul.-04 to 2020-Jul.30 , in cycle 3 , camera 1 . The second visit was observed as part of the TESS Guest Investigator Programs G03263 (PI: P. Plavchan), G03141 (PI: E. Newton), and G03273 (PI: L. Vega) in fast mode with a time sampling of $20 \mathrm{~s}$ compared to the 2 min time sampling of the first visit. We obtained the de-trended photometric time series from the Mikulski Archive for Space Telescopes (MAST) using the MAST astroquery tool ${ }^{1}$. Figure 1 shows the AU Mic PDCSAP ${ }^{2}$ flux in units of electrons-per-second $\left(\mathrm{e}^{-} \mathrm{s}^{-1}\right)$ as a function of time given in TESS Barycentric Julian Date (TBJD $=$ BJD -2457000.0$)$ for the two visits. Plavchan et al. (2020) have analyzed the same TESS data from the first visit only, and in the present paper we report for the first time an analysis including the more recent 2020 TESS data. The predicted times of the transits of AU Mic b and c as calculated from our ephemerides (see Sects. 5 and 6) are marked with vertical lines.

\section{Starspots}

As illustrated in Fig. 1, the TESS light curve of AU Mic shows a typical BY Draconis type of quasi-periodic variation due to starspots modulated by stellar rotation. The starspots evolve and, therefore, this modulation cannot be accurately modeled by a single periodic function. Thus, we treat this quasi-periodic modulation as a baseline "continuum" signal, where we model it by fitting a piece-wise fourth-order polynomial with iterative sigmaclipping. This approach works well when the ranges are carefully selected and inspected as follows. First, we can be certain that the polynomial function is a sufficiently accurate approximation

\footnotetext{
1 https://astroquery.readthedocs.io/en/latest/mast/ mast.html

2 Pre-search Data Conditioning SAP.
}

for the local baseline variation within each range. Then we avoid the edges of the ranges to include either a transit or a flare event. Finally, we avoid the inclusion of large gaps (when TESS did not deliver data) within the same range. The edges for all selected ranges are represented by the blue vertical dashed lines in Fig. 1.

This starspot model is removed from the data to model flares and planetary transits as explained in Sects. 4-6, which are further removed from the original data to fit the starspot modulation again. We repeat this procedure iteratively until the standard deviation of residuals is not improved by more than $1 \%$. We typically meet this criterion upon three to five iterations. The final starspot model is also shown in Fig. 1. In Appendix A, we present an independent measurement of the rotational period of AU Mic from the starspot modulation in the 2018 TESS data.

\section{Flares}

$\mathrm{AU}$ Mic is an active young star with intense flaring activity. We carry out an analysis of the flares in the TESS data to improve the constraints on the transit events. We consider the starspot-subtracted residuals as shown in Fig. 2. The flares are typically clusters of points lying above the noise level. First we detect peaks as possible candidate flares using the scipy.signal.find_peaks ${ }^{3}$ routine, where it finds local maxima via a simple comparison of neighbouring values. We set a minimum peak amplitude of $2.5 \sigma$ and a minimum horizontal distance between peaks of about $\sim 100 \mathrm{~min}$. Some flares in $\mathrm{AU}$ Mic are quite complex, while other flares occur before a previously initiated flare has ended. Therefore, we visually inspected all detected peaks to identify any possible additional peaks that have been missed by the find-peaks algorithm. After a few iterations, we identified a total of 324 flares in the whole TESS time series.

The times and amplitudes of detected peaks were adopted as initial values for the basis of performing a least-squares fit using the multi-flare model of Davenport et al. (2014), where each flare is represented by a two-phase model with a polynomial rise and an exponential decay. Figure 3 shows, as an example, the residual light curve and fit flares model for three days of flaring activity in AU Mic in both TESS visits. For each flare in the model, we fit the flare amplitude $\left(f_{\max }\right)$, the full width at half maximum (FWHM), and the time of maximum $t_{\text {peak }}$. The fit parameters for all flares are presented in Appendix B.

The total continuous monitoring time of AU Mic performed by TESS was estimated as $49.75 \mathrm{~d}$, where we have discounted the large gaps in the data. We detected a total of 324 flares, from which 316 have fit amplitudes above $F_{\max }>1 \sigma$, for $\sigma=161.5 \mathrm{e}^{-} \mathrm{s}^{-1}$. Thus, the AU Mic global occurrence rate of flares with amplitude above $f_{\max } \gtrsim 0.06 \%$ of the median stellar flux $\left(279544 \mathrm{e}^{-} \mathrm{s}^{-1}\right)$ is estimated as 6.35 flares per day or about 1 flare every $3.8 \mathrm{~h}$. Figure 4 shows the distribution of flares as function of flare amplitudes. We fit an exponential to the measured distribution and obtained an empirical model for the occurrence rate as function of flare amplitude of $f_{\mathrm{r}}=0.17 \mathrm{e}^{-4.0 f_{\max }} \mathrm{d}^{-1}$, with $f_{\max }$ in units of $10^{2} \mathrm{e}^{-} \mathrm{s}^{-1}$. We notice that the strong flares, which are more important energetically, have a much lower occurrence rate and therefore a low statistical significance in our analysis. Thus, this empirical relationship is only expected to hold for flares above the detection limit imposed by the noise and for flares with a statistically significant number of events observed

\footnotetext{
3 https://docs.scipy.org/doc/scipy/reference/ generated/scipy.signal.find_peaks.html
} 

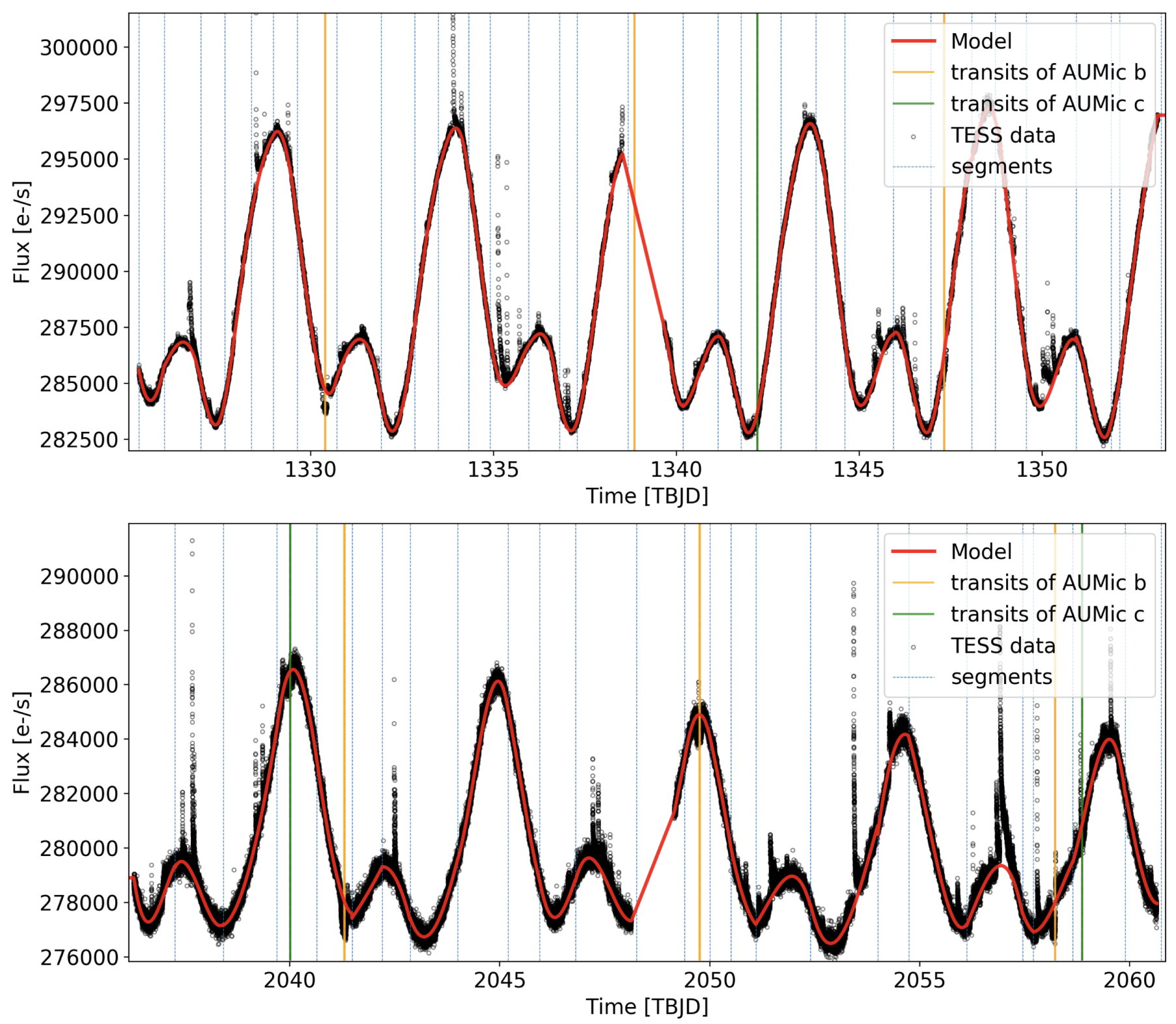

Fig. 1. TESS light curve of AU Mic. Top panel: data for the first visit (from 2018-Jul.-25 to 2018-Aug.-22). Bottom panel: data for the second visit (from 2020-Jul.-04 to 2020-Jul.-30). Black points are the PDCSAP flux and vertical solid lines show the times of planetary transits, as obtained in our analysis, for AU Mic b (in orange) and AU Mic c (in green). Blue vertical dashed lines show the knots of selected ranges for the piece-wise polynomial fit and the red line shows the starspot model.

in the TESS data, that is, those with amplitudes in the range of $0.06 \%<f_{\max }<1.5 \%$ of the star flux.

The transit duration of AU Mic b calculated by Plavchan et al. (2020) is about $3.5 \mathrm{~h}$ with transit depth of $0.26 \%$. Therefore, considering that a transit observation of $\mathrm{AU}$ Mic $\mathrm{b}$ requires an out-of-transit baseline of about $2 \mathrm{~h}$, it is most likely that any transit observation in AU Mic should be affected in some way by flare events. This shows the importance of modeling flares adequately to improve the constraints on the planetary parameters obtained by the transits.

\section{Transits of AU Mic b}

Three transits of AU Mic b occurred during the first TESS visit, but only two have been observed due to a communication problem with the spacecraft during the second transit (see
Plavchan et al. 2020). For the second visit, three other transits were expected, and indeed we were able to identify all of them. To analyze these five transits together, we start with the parameters of AU Mic b from Plavchan et al. (2020), referred to as "prior values" in Table 2, to remove the transit signal from the TESS light curve before fitting the starspot and flare models. Our transit model is calculated using the BATMAN toolkit by Kreidberg (2015), where we assume a circular orbit $(e=0)$. This assumption is in agreement with Plavchan et al. (2020), who did not detect any significant eccentricity from their analysis of the transits only, where the eccentricity would be slightly constrained by the duration of the transit. We adopt as priors the quadratic limb-darkening coefficients (LDC) from Claret (2018) with an arbitrary error of 0.1 , where we obtained the values calculated for the photometric system of TESS and those matching the closest stellar parameters to those of AU Mic (see Table 1). 

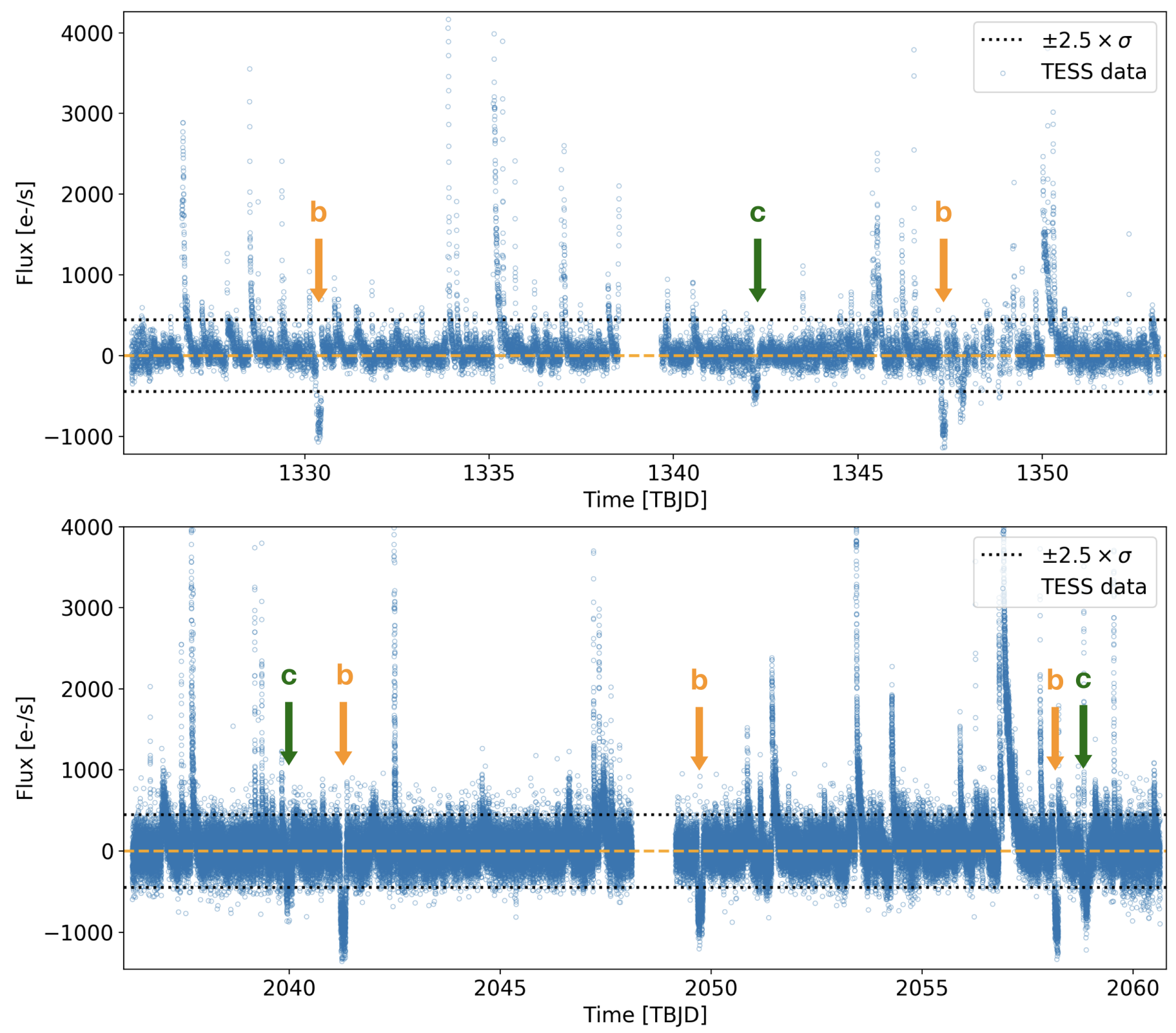

Fig. 2. TESS residual light curve of AU Mic. Top panel: residual light curve for the first TESS visit (2018-Jul.-25 to 2018-Aug.-22). Bottom panel: residual curve for the second visit (2020-Jul.-04 to 2020-Jul.-30). Blue circles show AU Mic TESS flux data after subtracting the starspot model and black dotted lines show $\pm 2.5 \sigma$ range around the starspot fit model, for $\sigma=162.5 \mathrm{e}^{-} \mathrm{s}^{-1}$. The flaring activity can be seen as positive groups of points above $+2.5 \sigma$ and the transits as negative groups of points below $-2.5 \sigma$. Our predicted times of transits for AU Mic b (in orange) and $\mathrm{AU}$ Mic c (in green) are marked with arrows.

The transits of AU Mic b, flares, and starspots are fit simultaneously using the non-linear least squares optimization (OLS) fit tool scipy.optimize.leastsq. As explained in Sect. 3 this procedure is run iteratively, where we first obtain an independent fit for each component of the model and then we set these values as initial guess to run an OLS analysis with all free parameters in the three components of the model. Then we consider the data in the ranges around the transits of AU Mic b to sample the posterior distributions of the transit parameters using the emcee Markov chain Monte Carlo (MCMC) package (ForemanMackey et al. 2013). The chain is set with 100 walkers and 30000 MCMC steps of which we discard the first 5000. The MCMC samples are presented in Fig. C.1, which show the chains reaching stability before the first 5000 discarded steps. The posterior distributions are illustrated in Fig. C.3. The best-fit values of the transit parameters are calculated as the medians of the posterior distribution with error bars defined by the $34 \%$ on each side of the median, all presented in Table 2.

Figure 5 shows the results of our analysis for each transit range separately, and the bottom right panel shows the flux normalized by the starspot and flare models for all transits together, and our best fit model along with the previous model of Plavchan et al. (2020) for comparison. The dispersion of residuals are $358,433,633,566$, and $588 \mathrm{ppm}$, for each respective epoch. The global dispersion is $573 \mathrm{ppm}$. Notice that our measured parameters of AU Mic b agree within $3 \sigma$ with the previous measurements by Plavchan et al. (2020) but with improved accuracy. Our measured planet-to-star radius ratio is slightly larger than that measured by Plavchan et al. (2020; see lower, right panel of Fig. 5). However, as described in Sect. 7, our derived effective 
Table 2. Transit fit parameters for planet AU Mic b obtained in our analysis from a simultaneous fit of the five transits observed by TESS as illustrated in Figs. 5 and C.3.

\begin{tabular}{lcc}
\hline \hline Parameter & Prior value & MCMC fit value \\
\hline Time of conjunction, $T_{0}[\mathrm{TBJD}]$ & $1330.38957_{-0.00068}^{+0.00015}$ & $1330.39051 \pm 0.00015$ \\
Orbital period, $P$ [d] & $8.46321 \pm 0.00004$ & $8.463000 \pm 0.000002$ \\
Normalized semi-major axis, $a / R_{\star}$ & $19.1_{-1.6}^{+1.8}$ & $19.1_{-0.4}^{+0.2}$ \\
Orbital inclination, $i$ [degree] & $89.5^{ \pm} 0.4$ & $89.5_{-0.3}^{+0.4}$ \\
Planet-to-star radius ratio, $R_{\mathrm{p}} / R_{\star}$ & $0.0514 \pm 0.0013$ & $0.0526_{-0.0003}^{+0.0000}$ \\
Linear limb dark. coef., $u_{0}$ & $\mathcal{N}(0.23,0.1)$ & $0.13 \pm 0.03$ \\
Quadratic limb dark. coef., $u_{1}$ & $\mathcal{N}(0.38,0.1)$ & $0.58 \pm 0.06$ \\
\hline
\end{tabular}
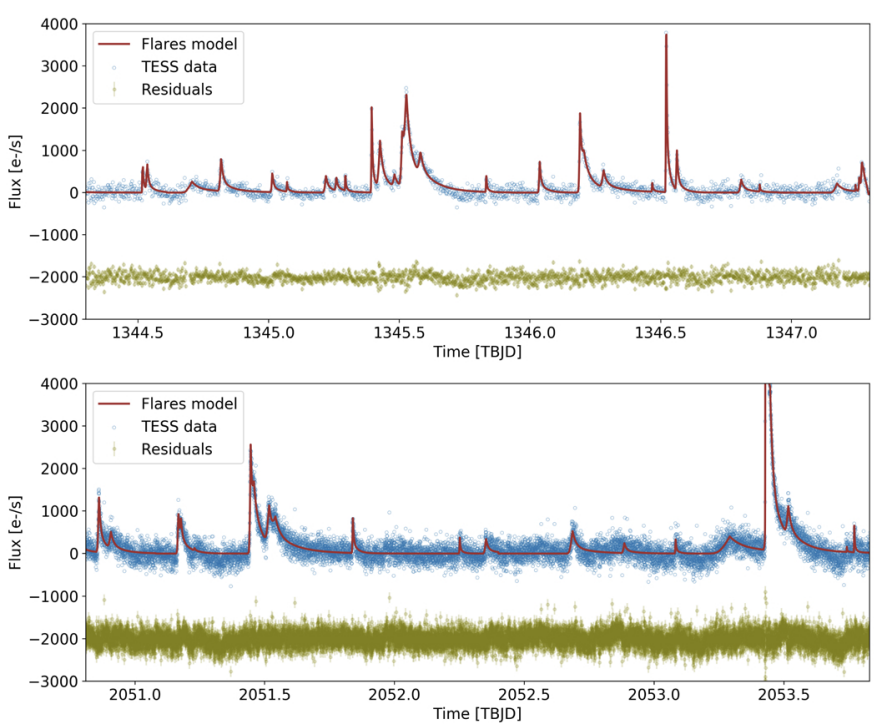

Fig. 3. TESS residual light curve of AU Mic between TBJD $=1344.3$ and $\mathrm{TBJD}=1347.3$ (top panel) and between $\mathrm{TBJD}=2050.8$ and TBJD $=2053.8$ (bottom panel). Blue circles show the flux after subtracting the starspot model, brown line shows the multi-flare best fit model. Green points show the residuals with an arbitrary offset for better visualization. The dispersion of residuals are $107 \mathrm{e}^{-} \mathrm{s}^{-1}$ and $178 \mathrm{e}^{-} \mathrm{s}^{-1}$ for data presented in top panel and bottom panel, respectively.

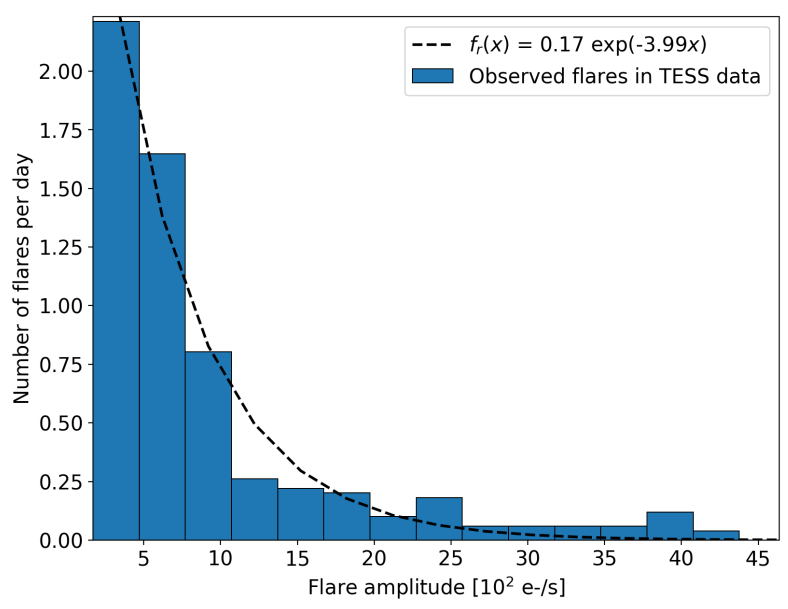

Fig. 4. Number of flares normalized by the total observed time of $49.75 \mathrm{~d}$ as function of flare amplitude. Dashed line shows the fit to an exponential decay as shown in the legend, where variable $x$ represents the flare amplitude. planetary radius of $1.05 \pm 0.04 R_{\mathrm{Nep}}$ is slightly smaller than the value $1.08 \pm 0.05 R_{\mathrm{Nep}}$ reported by Plavchan et al. (2020).

\section{Confirmation and characterization of planet AU Mic c}

An isolated candidate transit event at $T_{\mathrm{c}}=1342.22 \pm 0.03$ TBJD was identified in the 2018 TESS data by Plavchan et al. (2020), which was interpreted as a possible second planet in a $30 \pm 6-d$ orbit. We have searched for other transit-like events in the TESS data by looking at regions with systematic flux dips below the noise level. We identified three regions as potential transits including the one previously identified by Plavchan et al. (2020) (see Fig. 2). We notice that there is an additional region presenting a significant flux dip around TBJD $=1347.8$, which did not include as a possible transit. The 2018 TESS data after TBJD 1347.7 contain several gaps, which have an impact on the modeling of the baseline modulation by starspots as well as on the detection and modeling of flares. Therefore, we presume that the apparent flux dip in this region is more likely due to a misfit other than a transit. In addition the shape of that dip is different from that of the three features above.

We hypothesize that the three selected events are transits of the same candidate planet AU Mic c. In order to test this hypothesis, we first obtain an orbital period that is consistent with the three transits, that is, a period given by the slope of a linear fit to the ephemeris equation, $T_{\mathrm{c}}=T_{0}+P \times E$, with $T_{\mathrm{c}}$ as the times of conjunction measured independently for each event and $E$ as the corresponding epochs to each event, assumed to be 0,37 , and 38. We find an orbital period of $P=18.85895 \pm 0.00003 \mathrm{~d}$. However, we note that with this particular periodicity, only three events would occur during the TESS observations (see Fig. 1). The possibility of shorter alias periods is ruled out by the fact that TESS would have observed more transits of this object and we did not detect them. To test further our hypothesis, we performed an independent analysis of each event assuming an orbital period, first with an uniform prior of $P=\mathcal{U}(1,500) \mathrm{d}$ to explore a broad range of periods, and then with a normal prior of $P=\mathcal{N}(18.86,0.01)$ d, which explores a solution constrained by the previous knowledge imposed by our hypothesis. Analogously, the normalized semi-major axes are also first set with an uniform prior of $a / R_{\star}=\mathcal{U}(1,500)$ and then with a normal prior estimated using the Kepler's law, that is, $a / R_{\star}=\mathcal{N}(31.5,1.4)$. The priors for the times of conjunction are measured locally on each event, for example, for the first event, we obtained $T_{0}=\mathcal{N}(1342.23,0.01)$. The prior for the planet-to-star radius ratio is estimated from the square root of the average flux depth of the three events, assuming a conservative error, that 

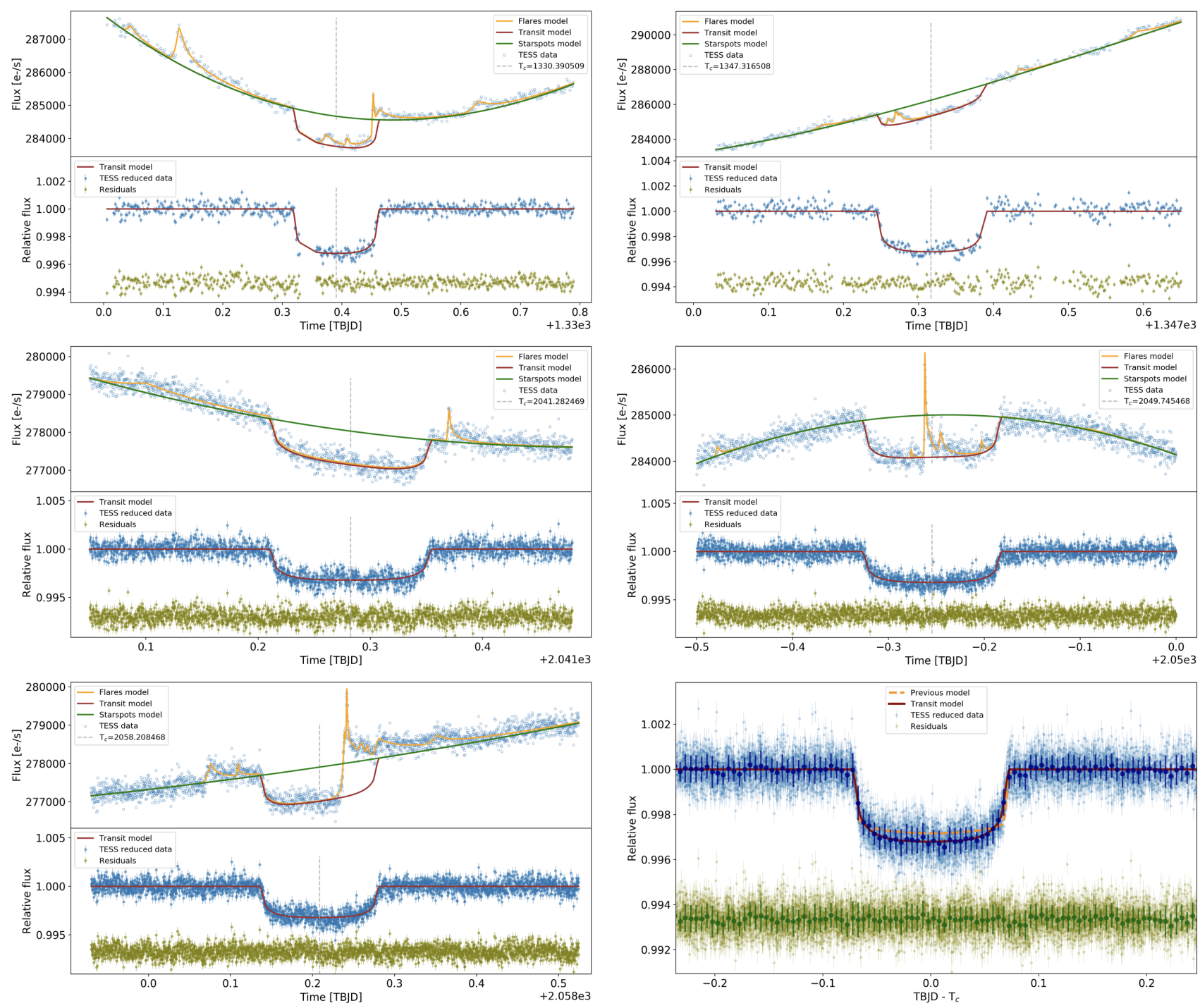

Fig. 5. Each panel shows the range around one of the three transits of AU Mic b observed by TESS. The top part of each panel shows the TESS data (blue circles) and the best-fit model for each component, as indicated in the legend. The bottom part in each panel shows the TESS data normalized by the starspot and flare model (blue points) and the best-fit transit model (red line). Vertical dashed lines show the central time of each transit. Bottom right panel: phase-folded light curve for all the data from the five transits (light blue points) and the binned data (dark blue points) with a bin size of $0.005 \mathrm{~d}$, our best fit model (red line), and the previous fit model of Plavchan et al. (2020) for comparison (orange dashed line). The residuals (green points) are displayed with an arbitrary offset.

is, $R_{\mathrm{p}} / R_{\star}=\mathcal{N}(0.04,0.01)$. The orbital inclination is considered with an uniform prior distribution of $i=\mathcal{U}\left(85^{\circ}, 90^{\circ}\right)$ and the limb darkening coefficients are fixed to the literature values presented in Table 1. In Appendix D, we present the results for this independent analysis of each transit. The posterior of all the transit parameters agree within $3 \sigma$ (see Table D.1), and the dispersion of residuals improves when we adopted the fit period of $P=\mathcal{N}(18.86,0.01) \mathrm{d}$ as prior, which supports our hypothesis that these three events were caused by the transits of the same planet AU Mic c. Finally, we perform a joint analysis of the three transits simultaneously, where we adopt the same priors as in the independent analysis above, except for the limb darkening coefficients, where we adopted a normal prior with the literature values and an arbitrary error of 1.0. We call attention to the fact that the limb darkening coefficients obtained in Sect. 5 could have been used as priors since both planets are transiting the same star. However, the two planets may transit the stellar disk in different regions, and since AU Mic is largely filled by starspots, the limb darkening may also be affected by the different temperatures of the transited regions in the photosphere. Table 3 presents the priors and fit parameters, while Fig. 6 shows the TESS data for the three transits and the bestfit model obtained from our analysis. As in Sect. 5, the MCMC samples and posterior distributions are illustrated in Figs. C.2 and C.4.

\section{Discussion}

We combine the transit parameters of AU Mic b and c presented in Tables 2 and 3 with the star parameters from Table 1 to calculate a number of derived parameters, which are presented in Table 4.

The planet-to-star radius ratio obtained in Sects. 5 and 6 are likely overestimated due to the presence of spots covering a significant fraction of the stellar photosphere. This is because the 
Table 3. Transit fit parameters for planet AU Mic c obtained in our analysis from a simultaneous fit of the three transits observed by TESS as illustrated in Figs. 6 and C.4.

\begin{tabular}{lcc}
\hline \hline Parameter & Prior value & MCMC fit value \\
\hline Time of conjunction, $T_{0}$ [TBJD] & $\mathcal{N}(1342.23,0.01)$ & $1342.2223 \pm 0.0005$ \\
Orbital period, $P$ [d] & $\mathcal{N}(18.86,0.01)$ & $18.859019_{-0.000016}^{+0.00016}$ \\
Normalized semi-major axis, $a / R_{\star}$ & $\mathcal{N}(31.5,1.4)$ & $29 \pm 3$ \\
Orbital inclination, $i$ [degree] & $\mathcal{U}(85,90)$ & $89.0_{-0.4}^{+0.5}$ \\
Planet-to-star radius ratio, $R_{\mathrm{p}} / R_{\star}$ & $\mathcal{N}(0.04,0.01)$ & $0.0418_{-0}^{+0.0010}$ \\
Linear limb dark. coef., $u_{0}$ & $\mathcal{N}(0.23,1.0)$ & $0.0_{-0.3}^{+0.2}$ \\
Quadratic limb dark. coef., $u_{1}$ & $\mathcal{N}(0.38,1.0)$ & $1.2_{-0.3}^{+0.4}$ \\
\hline
\end{tabular}
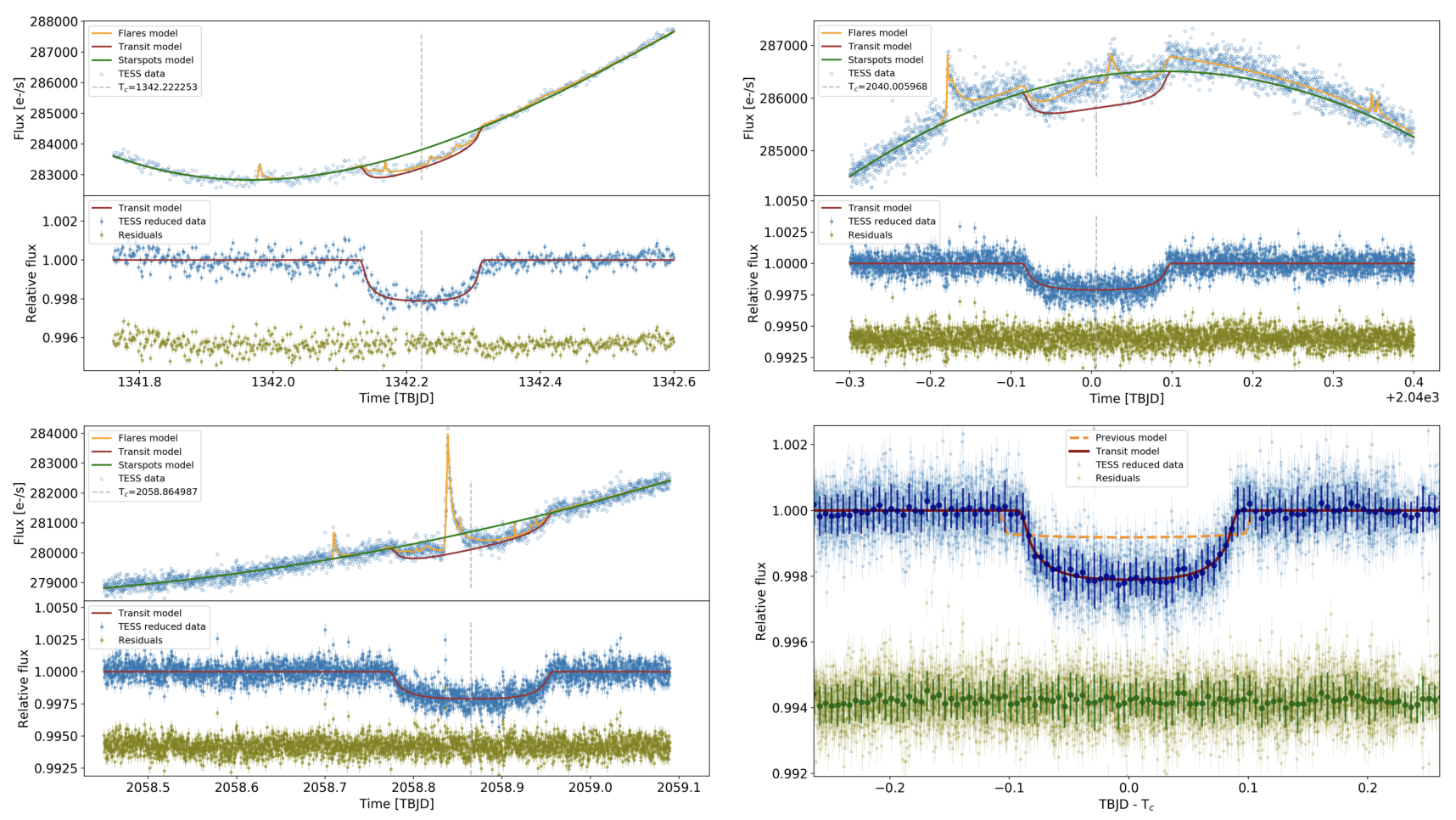

Fig. 6. Each panel shows the range around one of the three transits of AU Mic b observed by TESS. Details are the same as in Fig. 5, but here we present the results for the three TESS transits of AU Mic c. The dispersion of residuals are 370, 646, 609 ppm, for each respective epoch. The global dispersion is $609 \mathrm{ppm}$.

observed transit depth is likely smaller than the nominal depth that would be obtained for a completely unspotted photosphere. Therefore, an effective planet-to-star radius ratio can be obtained by applying a correction factor to the observed transit depth as in Eq. (1) of Rackham et al. (2018), that is,

$\left(\frac{R_{\mathrm{p}}}{R_{\star}}\right)_{\mathrm{eff}}^{2}=\left[1-f_{\text {spot }}\left(1-\frac{F_{\text {spot }}}{F_{\text {phot }}}\right)\right]\left(\frac{R_{\mathrm{p}}}{R_{\star}}\right)_{\mathrm{obs}}^{2}$

for $f_{\text {spot }}$ being the spot filling factor estimated at $20 \%$ based on magnetic activity (Klein et al. 2021) and $F_{\text {spot }} / F_{\text {phot }}$ being the flux fraction between the spots and the photosphere. The latter can be estimated assuming a blackbody flux for each of the two components, where the photospheric temperature is $T_{\text {phot }}=3700 \mathrm{~K}$ and the spots' temperature is estimated at $86 \%$ of the photospheric value in M1 stars (Rackham et al. 2018). The latter is in agreement with spectropolarimetric measurements of AU Mic by Berdyugina (2011). Our estimation for the spot-to-photosphere flux fraction integrated over the TESS band pass (between 60 and $1000 \mathrm{~nm}$ ) is $F_{\text {spot }} / F_{\text {phot }} \sim 46 \%$. Therefore, the effective radius for both transiting planets in the AU Mic system should be given by $R_{\mathrm{p}}^{\text {eff }}=0.94 R_{\mathrm{p}}^{\mathrm{obs}}$, that is, about $6 \%$ smaller than the measured radius.

In addition to the correction above, there is an uncertainty in the planet radius due to the rotational modulation by starspots and other photospheric heterogeneities, which implies a variable baseline flux. To account for this additional uncertainty, we considered the amplitude of the photospheric modulation in the AU Mic TESS light curve, which is about 5\%. This variability propagates an uncertainty on the order of $\sim \sqrt{0.05}$ to the planet radius measurements, which is included in the uncertainty values reported in Table 4.

Klein et al. (2021) recently measured a semi-amplitude of the radial velocity of the reflex motion caused by AU Mic b of $K_{\mathrm{b}}=8.5_{-2.2}^{+2.3} \mathrm{~m} \mathrm{~s}^{-1}$, providing an important constraint on the mass of this planet. We recalculated the planet density based on our radius measurement and obtained $\rho_{\mathrm{b}}=1.4 \pm 0.4 \mathrm{~g} \mathrm{~cm}^{-3}$. 
Table 4. Summary of the parameters of planets AU Mic b and AU Mic c.

\begin{tabular}{|c|c|c|c|}
\hline Parameter & Unit & AU Mic b & AU Mic c \\
\hline Time of conjunction & TBJD & $1330.39051 \pm 0.00015$ & $1342.2223 \pm 0.0005$ \\
\hline Orbital period & $\mathrm{d}$ & $8.463000 \pm 0.000002$ & $18.859019 \pm 0.000016$ \\
\hline Normalized semi-major axis, $a / R_{\star}$ & - & $19.1 \pm 0.3$ & $29 \pm 3$ \\
\hline Semi-major axis ${ }^{(\dagger)}$ & $\mathrm{au}$ & $0.0645 \pm 0.0013$ & $0.1101 \pm 0.0022$ \\
\hline Transit duration & $\mathrm{h}$ & $3.50 \pm 0.08$ & $4.5 \pm 0.8$ \\
\hline Orbital inclination & degree & $89.5 \pm 0.3$ & $89.0_{-0.4}^{+0.5}$ \\
\hline Impact parameter & $R_{\star}$ & $0.18 \pm 0.11$ & $0.51 \pm 0.21$ \\
\hline Eccentricity & - & 0 (FIXED) & 0 (FIXED) \\
\hline Planet-to-star radius ratio ${ }^{(\S)}, R_{\mathrm{p}} / R_{\star}$ & - & $0.0496 \pm 0.0007$ & $0.0395 \pm 0.0011$ \\
\hline Planet radius & $R_{\text {Jup }}$ & $0.371 \pm 0.016$ & $0.295 \pm 0.014$ \\
\hline Planet radius & $R_{\mathrm{Nep}}$ & $1.05 \pm 0.04$ & $0.84 \pm 0.04$ \\
\hline Planet radius & $R_{\oplus}$ & $4.07 \pm 0.17$ & $3.24 \pm 0.16$ \\
\hline Velocity semi-amplitude & $\mathrm{m} \mathrm{s}^{-1}$ & $8.5_{-2.2}^{+2.3(\S \S)}$ & $0.8<K_{\mathrm{c}}<9.5$ \\
\hline Planet mass & $M_{\text {Jup }}$ & $0.054 \pm 0.015^{(\S \S)}$ & $0.007<M_{\mathrm{c}}<0.079$ \\
\hline Planet mass & $M_{\mathrm{Nep}}$ & $1.00 \pm 0.27^{(\S \S)}$ & $0.13<M_{\mathrm{c}}<1.46$ \\
\hline Planet mass & $M_{\oplus}$ & $17 \pm 5^{(\S \S)}$ & $2.2<M_{\mathrm{c}}<25.0$ \\
\hline Planet density & $\mathrm{g} \mathrm{cm}^{-3}$ & $1.4 \pm 0.4$ & $0.4<\rho_{\mathrm{c}}<4.1$ \\
\hline Equilibrium temperature & $\mathrm{K}$ & $593 \pm 21$ & $454 \pm 16$ \\
\hline
\end{tabular}

Notes. ${ }^{(\dagger)}$ Semi-major axis derived from the fit period and the Kepler’s law; ${ }^{(\S \S)}$ radius values correspond to the effective radius, which is already corrected for spot coverage and contains an additional uncertainty due to rotational modulation, as explained in Sect. 7; ${ }^{(\$ \S)} \mathrm{Klein}$ et al. (2021).

To estimate a plausible mass range for AU Mic c, we considered a population of transiting exoplanets ${ }^{4}$ with radii within $1 \sigma$ of the measured radius of AU Mic c, as presented in Fig. 7. The median and median absolute deviation of the mass of exoplanets in this population is $0.043 \pm 0.036 M_{\text {Jup }}$. Therefore, the mass of AU Mic c is likely to lie in the range of $0.007 M_{\mathrm{Jup}}<$ $M_{\mathrm{c}}<0.079 M_{\mathrm{Jup}}$, implying a planet density in the range of $0.4 \mathrm{~g} \mathrm{~cm}^{-3}<\rho_{\mathrm{c}}<4.1 \mathrm{~g} \mathrm{~cm}^{-3}$. Several different scenarios are possible for the internal structure of this planet depending on its mass. We estimate the semi-amplitude of the induced radial velocity caused by $\mathrm{AU}$ Mic $\mathrm{c}$ in the star motion to be in the range of $0.8 \mathrm{~m} \mathrm{~s}^{-1}<K_{\mathrm{c}}<9.5 \mathrm{~m} \mathrm{~s}^{-1}$. A number of world-class spectrometers can currently achieve the necessary precision to detect such reflex motion. However, the intense stellar activity in AU Mic may represent a challenge for such measurement.

In order to analyze the stability of the orbital solution (Table 4), we performed a global frequency analysis (Laskar 1990,1993 ) in the vicinity of the best fit, in the same way as achieved for other planetary systems (e.g., Correia et al. 2005, 2010). The system is integrated on a regular $2 \mathrm{D}$ mesh of initial conditions, with varying semi-major axis and eccentricity of planet $\mathrm{c}$, while the other parameters are retained at their nominal values. We used the symplectic integrator SABA1064 of Farrés et al. (2013), with a step size of $5 \times 10^{-3} \mathrm{yr}$ and general relativity corrections. Each initial condition is integrated over $5 \mathrm{kyr}$, and a stability indicator is computed to be the variation in the measured mean motion over the two consecutive $2.5 \mathrm{kyr}$ intervals of time (for more details see Couetdic et al. 2010). For regular motion, there is no significant variation in the mean motion along the trajectory, while it can vary significantly for chaotic trajectories.

In Fig. 8, we show the wide vicinity of the nominal solution for two different configurations, one with $M_{\mathrm{c}}=25.0 M_{\oplus}$ (top) and another with $M_{\mathrm{c}}=2.2 M_{\oplus}$ (bottom), corresponding to the maximum and minimum masses of the outer planet, respectively.

4 Exoplanet parameters compiled from exoplanet.eu
The stability indicator is reported using a color index, where "red" represents the strongly chaotic trajectories and "dark-blue" shows the extremely stable ones. We observe that there are several islands of mean motion resonances nearby, however, the main difference is that when the mass of the outer planet is large, these resonances become unstable. The best fit nominal solution (vertical dotted line) is close to the 9:4 resonance, but outside, and so the nominal solution is in the stable zone for both mass values, as long as $e_{\mathrm{c}}<0.2$. We hence conclude that the AU Mic planetary system is stable and nearly circular.

The proximity to the 9:4 resonance could cause significant transit-timing variation (TTV). To measure the TTV for both planets, we run an MCMC analysis for each individual transit observed by TESS, where we obtained an independent measurement of the times of transits. The transit parameters are fixed to their best fit values, except $T_{\mathrm{c}}$, which is set with an uniform prior distribution of $\mathcal{U}\left(T_{\mathrm{c}}-0.005, T_{\mathrm{c}}+0.005\right)$, for $T_{\mathrm{c}}$ being the time of conjunction calculated from the fit ephemeris. We subtract $T_{\mathrm{c}}$ from the measured times of conjunction to obtain the TTVs as presented in Table 5. We detect no significant TTV in the TESS data. TTVs with amplitudes of one minute or more would have been likely detected in this dataset.

Using the nominal solution from Table 4, we generated the synthetic TTVs as in Hébrard et al. (2020), for the minimum and the maximum masses of the outer planet. In Fig. 9, we show the variations corresponding to each solution superimposed with the observational data (Table 5). For the outer planet, the amplitude of the TTV is around one minute for both mass choices. However, for the inner planet, the minimum mass produces TTV with an amplitude of only a few seconds, while for the maximum mass the amplitude can reach up to three minutes. Thus, the TTV of the inner planet can place constraints on the mass of the outer planet.

The precision and the number of photometric measurements currently available for the AU Mic system (Table 5) do not allow us to run an exhaustive search for a best fit solution, 


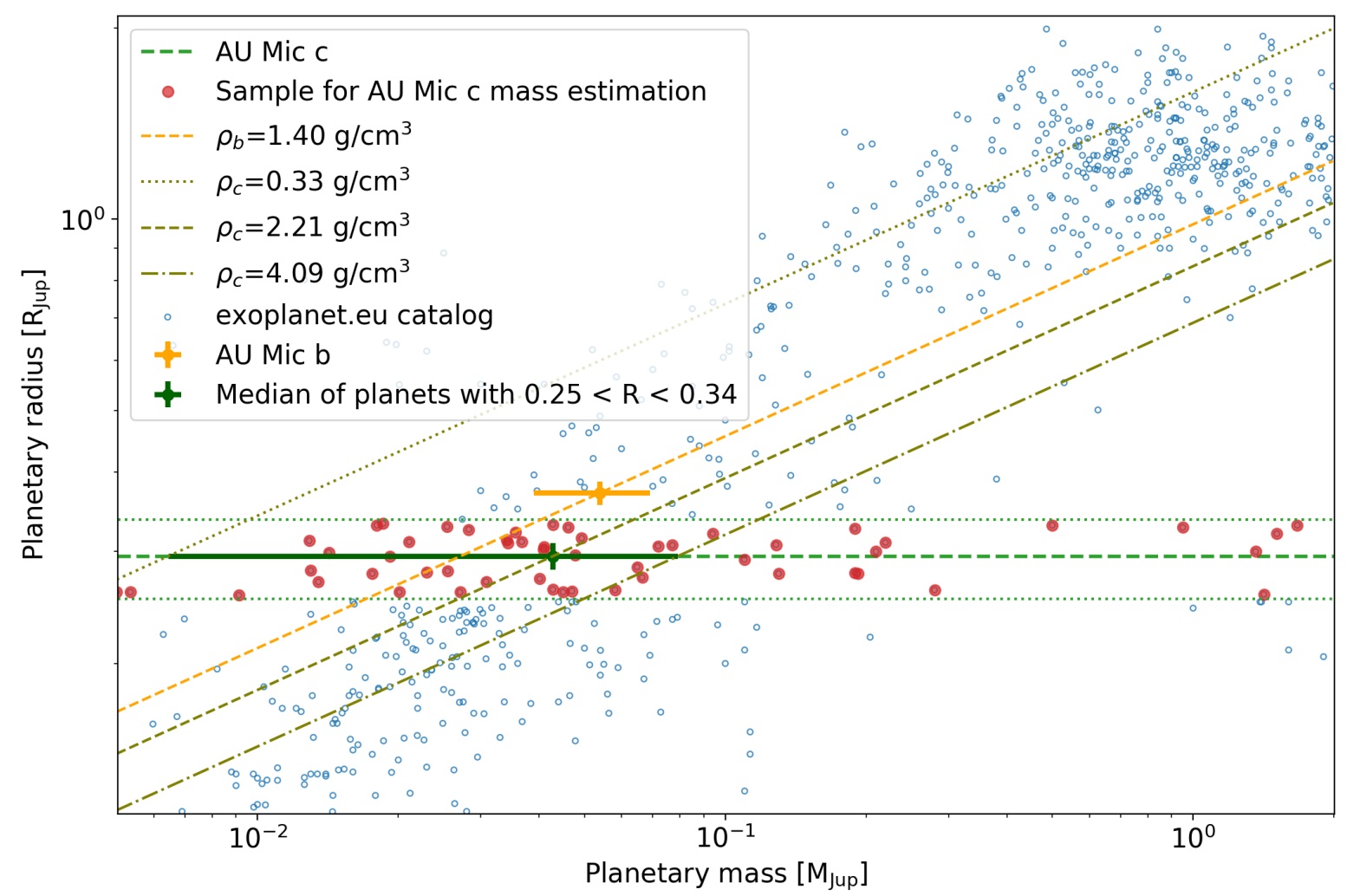

Fig. 7. Mass-radius diagram of planets. Blue circles show the known transiting exoplanets compiled from exoplanet. eu. The orange point represents AU Mic b and the light green horizontal dashed and dotted lines represent the AU Mic c radius measurement and $\pm 1 \sigma$ uncertainties. Dark green point represents the median of planets with radius within $1 \sigma$ of the measured radius of AU Mic c. Dashed orange line shows an isodensity line for the radius and mass of AU Mic b and the olive lines correspond to the iso-densities obtained for the radius of AU Mic $\mathrm{c}$ and for the lower limit (dotted), median value (dashed), and upper limit (dot-dashed) of the mass of AU Mic c, based on the exoplanet population with the same radii.

Table 5. Measurements of the transit-timing variation (TTV) for the transits of AU Mic b and c observed by TESS.

\begin{tabular}{cccc}
\hline \hline Planet & epoch & $T_{\mathrm{c}}(\mathrm{TBJD})$ & TTV $(\mathrm{s})$ \\
\hline $\mathrm{b}$ & 0 & $1330.39046 \pm 0.00016$ & $-32 \pm 17$ \\
$\mathrm{~b}$ & 2 & $1347.31646 \pm 0.00016$ & $+39 \pm 21$ \\
$\mathrm{~b}$ & 84 & $2041.28238 \pm 0.00026$ & $-41 \pm 25$ \\
$\mathrm{~b}$ & 85 & $2049.74538 \pm 0.00026$ & $+31 \pm 32$ \\
$\mathrm{~b}$ & 86 & $2058.20838 \pm 0.00026$ & $+27 \pm 27$ \\
\hline $\mathrm{c}$ & 0 & $1342.22432 \pm 0.00031$ & $-13 \pm 43$ \\
$\mathrm{c}$ & 37 & $2040.00697 \pm 0.00048$ & $-81 \pm 53$ \\
$\mathrm{c}$ & 38 & $2058.86596 \pm 0.00049$ & $+92 \pm 59$ \\
\hline
\end{tabular}

but they allow us to reduce the uncertainty in the mass of the outer planet. The non-detection of TTVs that are larger than one minute place the mass of the outer planet on the low side as the likelier assumption. In Fig. 9, we show the TTV corresponding to an outer companion with $M_{\mathrm{c}}=7.0 M_{\oplus}$. This mass generates TTVs with an amplitude around one minute corresponding to our upper limit. The observation of additional transits should help to resolve the present ambiguity in the outer planet mass.

Furthermore, we calculated the habitable zone (HZ) for $\mathrm{AU}$ Mic using the equations and data from Kopparapu et al. (2014), which gives an optimistic lower limit (recent Venus) at $0.25 \mathrm{au}$, and an upper limit (early Mars) at $0.64 \mathrm{au}$, with the runaway greenhouse limits $\left(M_{\mathrm{p}}=1 M_{\oplus}\right)$ ranging between 0.32 and $0.61 \mathrm{au}$. AU Mic b and $\mathrm{c}$ being at an orbital distance of
$0.0645 \pm 0.0013 \mathrm{au}$ and $0.1101 \pm 0.0022$ au are not in the HZ We estimate the equilibrium temperatures as in Heng \& Demory (2013) assuming an uniform heat redistribution and an arbitrary geometric albedo of 0.1 , which gives $T_{\text {eq,b }}=593 \pm 21 \mathrm{~K}$ and $T_{\text {eq,c }}=454 \pm 16 \mathrm{~K}$, for AU Mic b and c, respectively, showing that these warm Neptunes are indeed too hot to sustain water in liquid state. Moreover, the actual temperature for these planets could be much higher due to a possible greenhouse effect depending on their atmospheric composition and also due to an elevated internal temperature provided the young age of the system.

\section{Conclusions}

We presented an analysis of the photometric TESS observations of the active young M1 star AU Mic, where we model the rotational modulation by starspots, the flaring activity, and the planetary transits simultaneously. Our analysis delivered an estimation of the flare occurrence rate of 6.35 flares per day for flares with amplitudes in the range of $0.06 \%<f_{\max }<1.5 \%$ of the star flux. With such a flare rate it is important to model flaring activity to improve the constraints on the planetary parameters from transits. In our simple multi-flare model, we did not explore a detailed physical modeling of flares (e.g., as in Tilley et al. 2019) that could, in principle, be studied more extensively given the high quality and broad time coverage of these TESS observations. Our aim was to find an empirical description of flares that improves the constraints on the planetary parameters of AU Mic b and that increases the sensitivity for detecting and characterizing the second planet AU Mic c. 

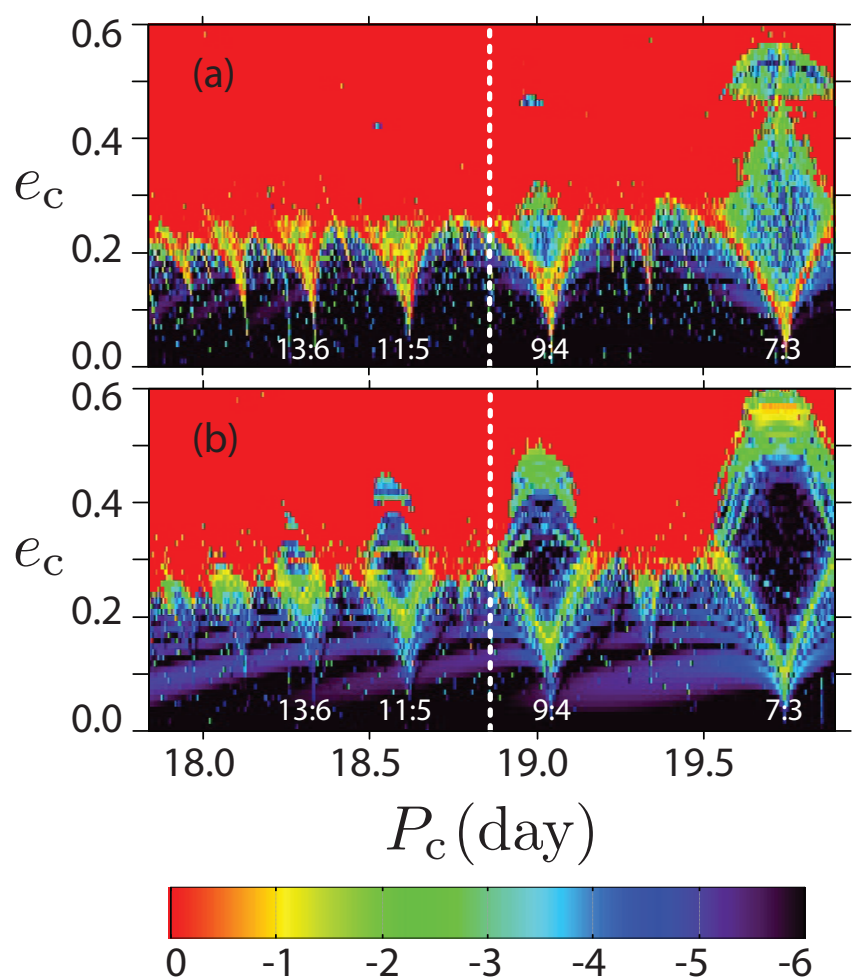

Fig. 8. Stability analysis of the AU Mic planetary system, around the best fit solution (Table 4), with $M_{\mathrm{c}}=25.0 M_{\oplus}(a)$, and $M_{\mathrm{c}}=2.2 M_{\oplus}(b)$. The phase space of the system is explored by varying the semi-major axis $a_{\mathrm{c}}$ (and thus the period $P_{\mathrm{c}}$ ) and eccentricity $e_{\mathrm{c}}$ of the outer planet; $e_{\mathrm{c}}$ is plotted as a function of $P_{\mathrm{c}}$ and not $a_{\mathrm{c}}$, as the uncertainty on $a_{\mathrm{c}}$ is larger. For the initial conditions, the step size is 0.01 in eccentricity, and $2 \times 10^{-5}$ au in semi-major axis. For each initial condition, the system is integrated over $5 \mathrm{kyr}$ and a stability criterion is derived with the frequency analysis of the mean longitude. The chaotic diffusion is measured by the variation in the mean motion. The color scale corresponds to the decimal logarithm of the variation of the mean motion (Correia et al. 2010). The "red" zone corresponds to highly unstable orbits, while the "dark-blue" region can be assumed to be stable on a billion-years timescale. The main resonances in the vicinity of the solution $(13: 6$, 11:5, 9:4, 7:3) are labeled. It should be noted that for the lower value of the mass $M_{\mathrm{c}}(b)$, the libration island is mostly stable while it is not the case for the larger value of $M_{\mathrm{c}}(a)$.

Our analysis of the five transits of AU Mic b provided measurements of the orbital period and time of conjunction, giving an improved ephemeris of $T_{\mathrm{c}}=2458330.39051 \pm 0.00015+$ $E \times 8.463000 \pm 0.000002 \mathrm{JD}$ for an accurate prediction of the times of future transits. Our detection of two new transits in the recent TESS observations have provided substantial evidence to confirm the second planet, that is, AU Mic c. We analyzed the three transits and obtained consistent results for the planetary parameters, which supports our hypothesis that these transits come from the same planet. Our MCMC analysis provided a determination of the planetary parameters for AU Mic c, delivering an ephemeris of $T_{\mathrm{c}}=2458342.2223 \pm$ $0.0005+E \times 18.859019_{-0.000015}^{+0.00001}$ JD for the times of transits with a transit duration of $4.5 \pm 0.8$ hours. The derived radius of $3.24 \pm 0.16 R_{\oplus}$ indicates that AU Mic c is a Neptune-size planet with an expected mass in the range of $2.2 M_{\oplus}<M_{\mathrm{c}}<25.0 M_{\oplus}$, estimated from the population of exoplanets of a similar size.

AU Mic b and c with an orbital period of $8.46 \mathrm{~d}$ and $18.86 \mathrm{~d}$ are near the 9:4 mean-motion resonance. Such a configuration is stable and could cause significant TTV, which we do not detect

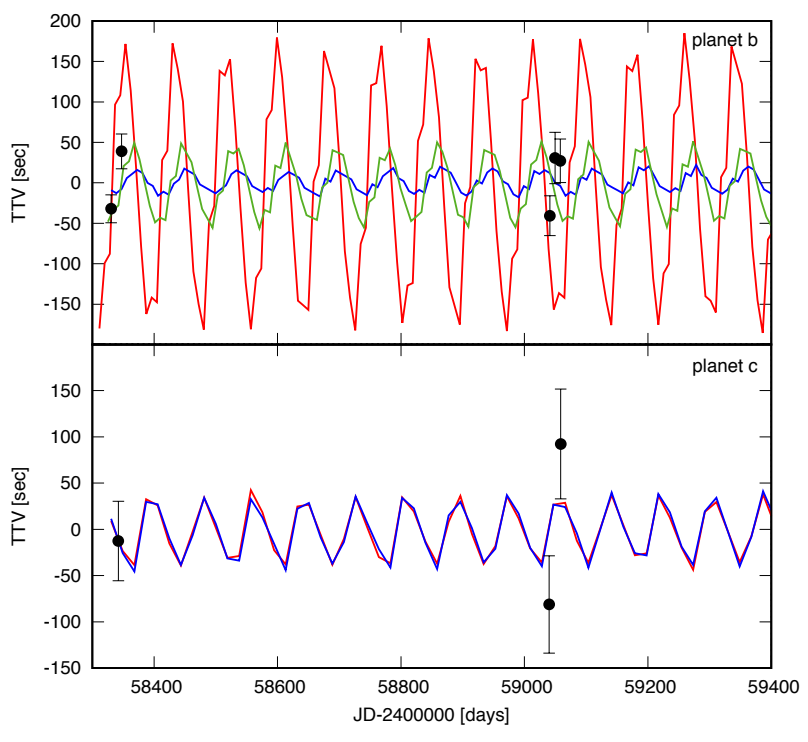

Fig. 9. Transit timing variations for AU Mic b (top) and AU Mic c (bottom). The colored lines correspond to synthetic data obtained with the best fit solution (Table 4) using different values for the mass of the outer planet: $M_{\mathrm{c}}=2.2 M_{\oplus}$ (blue), $M_{\mathrm{c}}=7.0 M_{\oplus}$ (green), and $M_{\mathrm{c}}=25.0 M_{\oplus}$ (red). The dots correspond to the observed TTVs. (Table 5).

with the current dataset. Still, that non-detection provides an upper limit to the mass of AU Mic c of $M_{\mathrm{c}}<7 M_{\oplus}$, implying an upper limit to the planet density of $\rho_{\mathrm{c}}<1.3 \mathrm{~g} \mathrm{~cm}^{-3}$. This suggests that both planets are likely inflated, as it should be expected for young planets (e.g., Helled et al. 2020). Therefore, these planets are interesting targets for atmospheric characterization by transmission spectroscopy.

The interactions between the planets coupled in a near 9:4 mean-motion resonance and the debris disk surrounding the star are to be the subject of subsequent studies. High-resolution images have revealed that the disk has a complex structure with small-sized substructures, such as "feature A" at a dozen astronomical units showing a "loop-like" morphology (Wisniewski et al. 2019). As for the case of $\beta$ Pictoris, it has been shown that the planets can sculpt the disk morphology through the gravitational interaction between planets and the debris disk parents bodies at far distances (Lecavelier Des Etangs et al. 1996; Augereau et al. 2001), particularly through resonance mechanisms in young systems that are still evolving (Lecavelier Des Etangs 1998). The impact of the presence of AU Mic b and AU Mic $\mathrm{c}$ on the dust disk deserves further analysis.

Acknowledgements. We acknowledge funding from the French National Research Agency (ANR) under contract number ANR-18-CE31-0019 (SPlaSH). This paper includes data collected with the TESS mission, obtained from the MAST data archive at the Space Telescope Science Institute (STScI). In particular, the TESS data secured in 2020 are part of the TESS Guest Investigator Programs G03263 (PI: P. Plavchan), G03141 (PI: E. Newton), and G03273 (PI: L. Vega). Together with other data and analyses of that particularly interesting system, that will be the subjects of forthcoming papers by Cale et al., Collins et al., El Mufti et al., Gilbert et al., Wittrock et al. Funding for the TESS mission is provided by the NASA Explorer Program. STScI is operated by the Association of Universities for Research in Astronomy, Inc., under NASA contract NAS 5-26555. We thank the TESS Team members for making available the extremely accurate photometric data they obtained. We acknowledge support by CFisUC projects (UIDB/04564/2020 and UIDP/04564/2020), GRAVITY (PTDC/FIS-AST/7002/2020), ENGAGE SKA (POCI-01-0145-FEDER-022217), and PHOBOS (POCI-01-0145-FEDER-029932), funded by COMPETE 2020 and FCT, Portugal. This work benefited from HPC resources of MesoPSL financed by the Region Ile de France and the project Equip@Meso (reference ANR-10-EQPX-29-01) of the programme Investissements d'Avenir. 
E. Martioli et al.: New constraints on the young planetary system AU Mic

\section{References}

Augereau, J. C., Nelson, R. P., Lagrange, A. M., Papaloizou, J. C. B., \& Mouillet, D. 2001, A\&A, 370, 447

Berdyugina, S. V. 2011, ASP Conf. Ser., 437, 219

Bonfils, X., Delfosse, X., Udry, S., et al. 2013, A\&A, 549, A109

Claret, A. 2018, A\&A, 618, A20

Correia, A. C. M., Udry, S., Mayor, M., et al. 2005, A\&A, 440, 751

Correia, A. C. M., Couetdic, J., Laskar, J., et al. 2010, A\&A, 511, A21

Couetdic, J., Laskar, J., Correia, A. C. M., Mayor, M., \& Udry, S. 2010, A\&A, 519, A 10

Davenport, J. R. A., Hawley, S. L., Hebb, L., et al. 2014, ApJ, 797, 122

Donati, J. F., Kouach, D., Moutou, C., et al. 2020, MNRAS, 498, 5684

Farrés, A., Laskar, J., Blanes, S., et al. 2013, Celest. Mech. Dyn. Astron., 116, 141

Foreman-Mackey, D., Hogg, D. W., Lang, D., \& Goodman, J. 2013, PASP, 125, 306

Gaia Collaboration 2018, VizieR Online Data Catalog: I/345

Hébrard, G., Díaz, R. F., Correia, A. C. M., et al. 2020, A\&A, 640, A32

Helled, R., Nettelmann, N., \& Guillot, T. 2020, Space Sci. Rev., 216, 38

Heng, K., \& Demory, B.-O. 2013, ApJ, 777, 100

Hirano, T., Krishnamurthy, V., Gaidos, E., et al. 2020, ApJ, 899, L13

Hsu, D. C., Ford, E. B., \& Terrien, R. 2020, MNRAS, 498, 2249

Kalas, P., Liu, M. C., \& Matthews, B. C. 2004, Science, 303, 1990
Klein, B., Donati, J.-F., Moutou, C., et al. 2021, MNRAS, 502, 188

Kopparapu, R. K., Ramirez, R. M., SchottelKotte, J., et al. 2014, ApJ, 787, L29

Kreidberg, L. 2015, PASP, 127, 1161

Laskar, J. 1990, Icarus, 88, 266

Laskar, J. 1993, Phys. D Nonlinear Phenomena, 67, 257

Lecavelier Des Etangs, A. 1998, A\&A, 337, 501

Lecavelier Des Etangs, A., Vidal-Madjar, A., \& Ferlet, R. 1996, A\&A, 307, 542

Mamajek, E. E., \& Bell, C. P. M. 2014, MNRAS, 445, 2169

Martioli, E., Hébrard, G., Moutou, C., et al. 2020, A\&A, 641, L1

Palle, E., Oshagh, M., Casasayas-Barris, N., et al. 2020, A\&A, 643, A25

Plavchan, P., Barclay, T., Gagné, J., et al. 2020, Nature, 582, 497

Rackham, B. V., Apai, D., \& Giampapa, M. S. 2018, ApJ, 853, 122

Ricker, G. R., Winn, J. N., Vanderspek, R., et al. 2015, J. Astron. Teles. Instrum. Syst., 1, 014003

Robinson, R. D., Linsky, J. L., Woodgate, B. E., \& Timothy, J. G. 2001, ApJ, 554,368

Stellingwerf, R. F. 1978, ApJ, 224, 953

Tilley, M. A., Segura, A., Meadows, V., Hawley, S., \& Davenport, J. 2019, Astrobiology, 19, 64

Torres, C. A. O., Ferraz Mello, S., \& Quast, G. R. 1972, Astrophys. Lett., 11, 13

White, R. J., Schaefer, G., Ten Brummelaar, T., et al. 2015, Am. Astron. So. Meeting Abs., 225, 348.12

Wisniewski, J. P., Kowalski, A. F., Davenport, J. R. A., et al. 2019, ApJ, 883, L8 


\section{Appendix A: Star rotation}

In this appendix, we present an independent measurement of the star rotation period of AU Mic.

Starspots in AU Mic have a life time sufficiently large that the overall spot pattern did not change significantly during the 2018 TESS observations in the first visit. As one can see in the bottom panel of Fig. 1, there has been a more significant change in the spot pattern during the second visit of TESS, where the amplitude of the cyclic flux variations decreases with time. For this reason, we did not include the second visit data to measure the rotation period. Flares and planetary transits are removed from the original data using the models computed in Sects. 4-6. We apply the phase dispersion minimization (PDM) method inspired by the work of Stellingwerf (1978), where we calculate the phase curve for several values of trial rotation periods ranging from $4.85 \mathrm{~d}$ to $4.88 \mathrm{~d}$ in steps of $0.0001 \mathrm{~d}$. The rotation period of $P_{\mathrm{r}}=4.865 \mathrm{~d}$ measured by Torres et al. (1972)

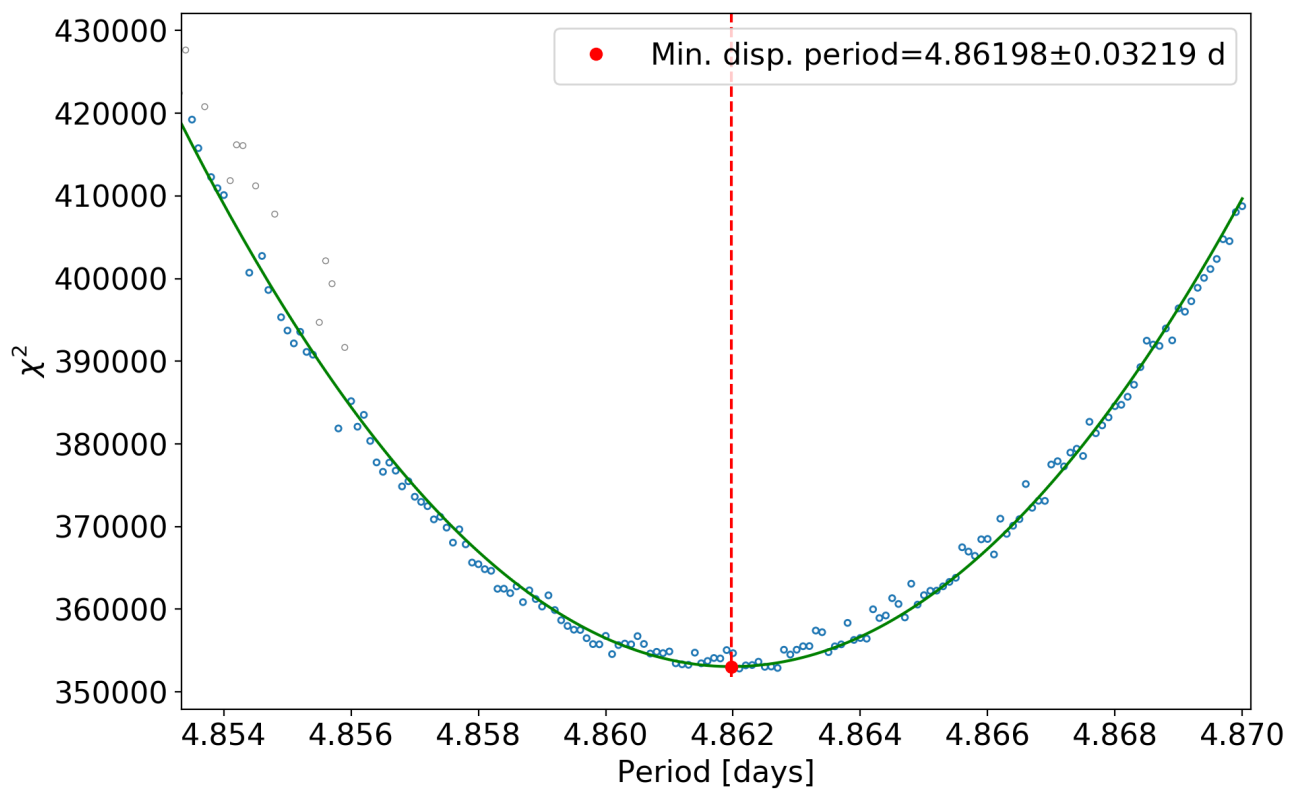

Fig. A.1. Rotation period versus chisquare for the reduced light curve of AU Mic after removing flares and planetary transits. Red dashed line shows the minimum $\chi^{2}$ at $P=4.862 \pm 0.032 \mathrm{~d}$ obtained by a parabolic fit (green line).

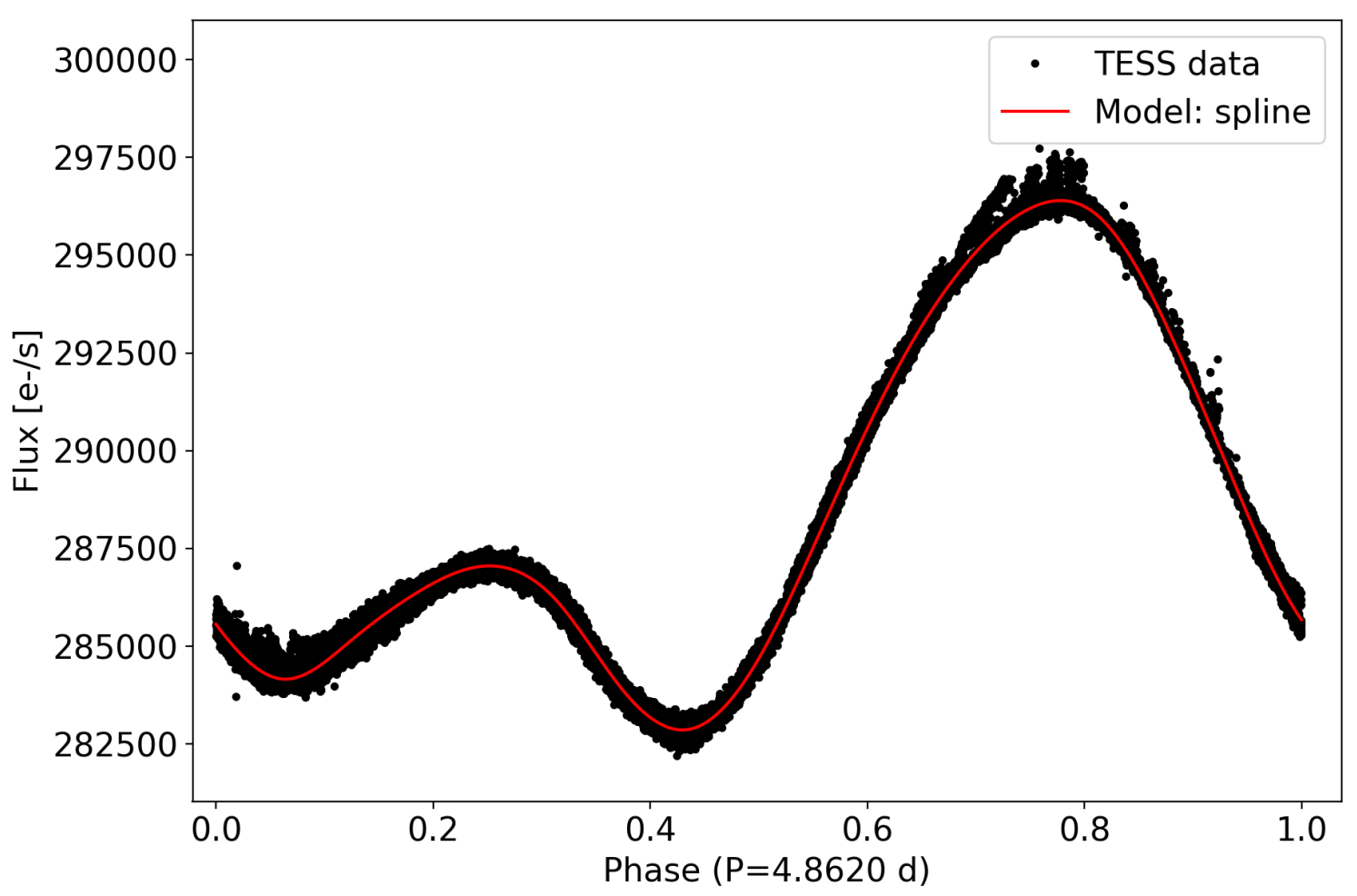

Fig. A.2. Phased light curve of AU Mic using our measured rotation period of 4.862 d. Black points show the 2018 TESS (first visit only) fluxes after removing the flares and transits best fit models, and red line shows the best fit cubic spline model to the phased data. 
was considered as initial guess to define our search range. For each trial period, we fit a cubic spline with 15 knots and calculate the chi-square by $\chi^{2}=\sum\left(F_{\mathrm{i}}-F_{\mathrm{c}}\right)^{2} / \sigma_{\mathrm{i}}^{2}$, for $F_{\mathrm{i}}$ being the flux after removing flares and transits, $F_{\mathrm{c}}$ being the fit model, and $\sigma_{\mathrm{i}}$ being the flux uncertainty given by the TESS pipeline. The results are presented in Fig. A.1, where we find a minimum $\chi^{2}$ at $P_{\mathrm{r}}=4.862 \pm 0.032 \mathrm{~d}$, which is consistent but less accurate than the rotation period of $4.863 \pm 0.010 \mathrm{~d}$ obtained by Plavchan et al. (2020) from the same data set. The resulting phase curve and best fit model are presented in Fig. A.2. The final dispersion of residuals is $265 \mathrm{e}^{-} \mathrm{s}^{-1}$, which is more than twice the dispersion of residuals from the fit to the time series, that is, $99 \mathrm{e}^{-} \mathrm{s}^{-1}$ for the data from the first visit of TESS only. This discrepancy is likely due to the evolution or drifts of starspots during the several rotation cycles covered in the first visit and perhaps also due to differential rotation (Klein et al. 2021), which could generate a dispersion in the phase diagram since starspots at different latitudes are modulated by different periods.

Assuming the radius of AU Mic measured by interferometry of $R_{\star}=0.75 \pm 0.03 R_{\odot}$ (White et al. 2015) and the rotation period of $P_{\mathrm{r}}=4.862 \pm 0.032 \mathrm{~d}$, we calculate the velocity at the equator as $v_{\text {eq }}=2 \pi R_{\star} / P_{\mathrm{r}}=7.8 \pm 0.3 \mathrm{~km} \mathrm{~s}^{-1}$. This gives a maximum value of the projected velocity of $v_{\mathrm{eq}} \sin \left(i_{\star}\right)<8.1 \mathrm{~km} \mathrm{~s}^{-1}$, for $i_{\star}$ being the inclination angle of the rotation axis of the star with respect to the line of sight.

\section{Appendix B: Flares fit parameters}

Table B.1. Flares fit parameters.

\begin{tabular}{rcrr}
\hline \hline $\begin{array}{c}\text { Flare } \\
\text { index }\end{array}$ & $\begin{array}{c}t_{\text {peak }} \\
\text { TBJD }\end{array}$ & $\begin{array}{r}\text { Amplitude } \\
\mathrm{e}^{-} \mathrm{s}^{-1}\end{array}$ & $\begin{array}{r}F W H \\
\mathrm{~min}\end{array}$ \\
\hline 1 & 1325.5892 & 427.5 & 31.84 \\
2 & 1325.7407 & 413.9 & 2.12 \\
3 & 1326.1596 & 284.5 & 16.06 \\
4 & 1326.2210 & 299.1 & 1.58 \\
5 & 1326.3398 & 167.0 & 44.57 \\
6 & 1326.3683 & 309.0 & 18.73 \\
7 & 1326.5566 & 235.3 & 6.42 \\
8 & 1326.6753 & 2121.5 & 20.65 \\
9 & 1326.6978 & 2553.5 & 41.60 \\
10 & 1326.7152 & 643.7 & 30.53 \\
11 & 1326.8131 & 407.7 & 17.67 \\
12 & 1327.1280 & 182.7 & 7.49 \\
13 & 1327.2165 & 710.9 & 40.91 \\
14 & 1327.3085 & 234.8 & 11.85 \\
15 & 1327.4389 & 475.8 & 11.59 \\
16 & 1327.4702 & 618.6 & 6.97 \\
17 & 1327.5782 & 154.6 & 81.53 \\
18 & 1327.8975 & 1430.4 & 8.48 \\
19 & 1327.9450 & 405.8 & 100.61 \\
20 & 1327.9970 & 259.6 & 35.31 \\
21 & 1328.1461 & 537.6 & 10.28 \\
22 & 1328.2454 & 323.7 & 7.80 \\
23 & 1328.5056 & 7272.7 & 7.27 \\
24 & 1328.7362 & 1838.0 & 3.24 \\
25 & 1329.0420 & 301.9 & 17.15 \\
26 & 1329.0900 & 378.9 & 14.88 \\
27 & 1329.3240 & 689.1 & 13.58 \\
28 & 1329.3803 & 2870.0 & 7.23 \\
29 & 1329.4333 & 861.4 & 13.85 \\
\hline & & & \\
& & &
\end{tabular}

Table B.1. continued.

\begin{tabular}{|c|c|c|c|}
\hline $\begin{array}{l}\text { Flare } \\
\text { index }\end{array}$ & $\begin{array}{c}t_{\text {peak }} \\
\text { TBJD } \\
\end{array}$ & $\begin{array}{r}\text { Amplitude } \\
\mathrm{e}^{-} \mathrm{s}^{-1} \\
\end{array}$ & $\begin{array}{r}F W H M \\
\min \\
\end{array}$ \\
\hline 30 & 1329.4581 & 168.4 & 23.54 \\
\hline 31 & 1330.0446 & 221.5 & 10.54 \\
\hline 32 & 1330.1271 & 1112.7 & 25.56 \\
\hline 33 & 1330.3750 & 338.9 & 36.00 \\
\hline 34 & 1330.4089 & 295.2 & 5.27 \\
\hline 35 & 1330.4475 & 184.4 & 71.83 \\
\hline 36 & 1330.4524 & 1517.1 & 4.97 \\
\hline 37 & 1330.6290 & 308.9 & 41.26 \\
\hline 38 & 1330.8089 & 793.3 & 37.58 \\
\hline 39 & 1330.9154 & 542.9 & 41.27 \\
\hline 40 & 1331.3989 & 576.3 & 37.30 \\
\hline 41 & 1331.4522 & 383.9 & 45.27 \\
\hline 42 & 1331.8229 & 1486.5 & 3.80 \\
\hline 43 & 1332.0069 & 178.2 & 20.97 \\
\hline 44 & 1332.1019 & 195.6 & 23.99 \\
\hline 45 & 1332.3460 & 536.7 & 9.86 \\
\hline 46 & 1332.4868 & 371.5 & 105.57 \\
\hline 47 & 1332.8700 & 299.1 & 12.73 \\
\hline 48 & 1333.0207 & 504.1 & 6.85 \\
\hline 49 & 1333.1734 & 617.6 & 22.72 \\
\hline 50 & 1333.7962 & 288.6 & 36.04 \\
\hline 51 & 1333.8896 & 5881.7 & 17.38 \\
\hline 52 & 1333.8961 & 1535.9 & 9.14 \\
\hline 53 & 1333.9019 & 1693.0 & 1.99 \\
\hline 54 & 1334.1192 & 2089.1 & 13.44 \\
\hline 55 & 1334.3769 & 811.2 & 5.82 \\
\hline 56 & 1334.3925 & 442.6 & 5.83 \\
\hline 57 & 1334.4750 & 172.1 & 61.01 \\
\hline 58 & 1334.6162 & 527.2 & 9.53 \\
\hline 59 & 1334.6378 & 223.0 & 8.21 \\
\hline 60 & 1335.1163 & 11469.8 & 13.44 \\
\hline 61 & 1335.1293 & 3267.2 & 11.25 \\
\hline 62 & 1335.1639 & 1568.5 & 119.59 \\
\hline 63 & 1335.3617 & 12798.6 & 5.00 \\
\hline 64 & 1335.7034 & 3266.6 & 4.68 \\
\hline 65 & 1335.7127 & 2214.6 & 0.65 \\
\hline 66 & 1335.7684 & 206.0 & 16.34 \\
\hline 67 & 1335.8508 & 202.2 & 17.09 \\
\hline 68 & 1336.1992 & 434.9 & 20.97 \\
\hline 69 & 1336.2313 & 1054.6 & 6.61 \\
\hline 70 & 1336.2723 & 246.9 & 6.35 \\
\hline 71 & 1336.3060 & 330.1 & 6.64 \\
\hline 72 & 1336.5145 & 506.0 & 4.03 \\
\hline 73 & 1336.5422 & 447.8 & 25.73 \\
\hline 74 & 1336.6280 & 384.5 & 6.94 \\
\hline 75 & 1336.9576 & 2572.0 & 9.42 \\
\hline 76 & 1337.0216 & 1119.7 & 9.59 \\
\hline 77 & 1337.0341 & 2277.9 & 21.60 \\
\hline 78 & 1337.4779 & 681.6 & 8.05 \\
\hline 79 & 1337.6259 & 473.3 & 3.50 \\
\hline 80 & 1337.7419 & 355.1 & 16.21 \\
\hline 81 & 1337.9638 & 346.6 & 18.35 \\
\hline 82 & 1338.0760 & 215.2 & 4.12 \\
\hline 83 & 1338.1343 & 190.1 & 7.34 \\
\hline 84 & 1338.2252 & 1050.7 & 11.79 \\
\hline 85 & 1338.2467 & 480.7 & 79.62 \\
\hline 86 & 1338.4465 & 1526.2 & 2.42 \\
\hline 87 & 1338.5024 & 1309.5 & 6.00 \\
\hline 88 & 1338.5213 & 2095.5 & 18.37 \\
\hline
\end{tabular}

A177, page 13 of 19 
Table B.1. continued.

\begin{tabular}{|c|c|c|c|}
\hline $\begin{array}{l}\text { Flare } \\
\text { index }\end{array}$ & $\begin{array}{c}t_{\text {peak }} \\
\text { TBJD }\end{array}$ & $\begin{array}{r}\text { Amplitude } \\
\mathrm{e}^{-} \mathrm{s}^{-1}\end{array}$ & $\begin{array}{r}F W H M \\
\text { min } \\
\end{array}$ \\
\hline 89 & 1339.7341 & 200.4 & 9.29 \\
\hline 90 & 1339.7816 & 466.4 & 34.29 \\
\hline 91 & 1339.8383 & 1029.1 & 10.20 \\
\hline 92 & 1340.5251 & 831.2 & 37.34 \\
\hline 93 & 1340.6133 & 399.3 & 26.63 \\
\hline 94 & 1340.9817 & 262.2 & 12.08 \\
\hline 95 & 1341.3729 & 390.8 & 34.20 \\
\hline 96 & 1341.9796 & 651.3 & 4.16 \\
\hline 97 & 1342.1527 & 234.9 & 33.97 \\
\hline 98 & 1342.1668 & 1938.2 & 0.72 \\
\hline 99 & 1342.2354 & 224.3 & 12.48 \\
\hline 100 & 1342.2719 & 209.6 & 18.33 \\
\hline 101 & 1342.2962 & 365.4 & 1.86 \\
\hline 102 & 1342.4018 & 122.2 & 39.11 \\
\hline 103 & 1342.6043 & 421.3 & 4.31 \\
\hline 104 & 1342.6336 & 255.9 & 24.70 \\
\hline 105 & 1343.5102 & 1152.4 & 10.70 \\
\hline 106 & 1343.9443 & 221.4 & 67.41 \\
\hline 107 & 1344.0673 & 308.4 & 8.81 \\
\hline 108 & 1344.2179 & 185.1 & 18.45 \\
\hline 109 & 1344.5174 & 1246.6 & 3.87 \\
\hline 110 & 1344.5350 & 699.4 & 11.15 \\
\hline 111 & 1344.7107 & 272.2 & 88.42 \\
\hline 112 & 1344.8182 & 782.1 & 18.63 \\
\hline 113 & 1345.0144 & 474.4 & 14.07 \\
\hline 114 & 1345.0717 & 405.8 & 3.72 \\
\hline 115 & 1345.2198 & 425.1 & 23.96 \\
\hline 116 & 1345.2599 & 296.4 & 23.24 \\
\hline 117 & 1345.3944 & 2205.4 & 6.26 \\
\hline 118 & 1345.4257 & 1232.8 & 24.61 \\
\hline 119 & 1345.4816 & 329.7 & 24.13 \\
\hline 120 & 1345.5112 & 1299.7 & 19.20 \\
\hline 121 & 1345.5269 & 1908.0 & 31.64 \\
\hline 122 & 1345.5806 & 512.1 & 39.17 \\
\hline 123 & 1345.8324 & 526.4 & 3.90 \\
\hline 124 & 1346.0371 & 756.7 & 11.13 \\
\hline 125 & 1346.1915 & 1815.8 & 11.52 \\
\hline 126 & 1346.2062 & 622.6 & 42.72 \\
\hline 127 & 1346.2819 & 404.2 & 25.73 \\
\hline 128 & 1346.4695 & 1355.0 & 0.72 \\
\hline 129 & 1346.5211 & 5102.5 & 4.92 \\
\hline 130 & 1346.5619 & 1014.5 & 9.39 \\
\hline 131 & 1346.8081 & 311.1 & 21.61 \\
\hline 132 & 1346.8791 & 221.4 & 2.58 \\
\hline 133 & 1347.1743 & 224.4 & 49.74 \\
\hline 134 & 1347.2600 & 436.4 & 7.55 \\
\hline 135 & 1347.2696 & 773.2 & 7.27 \\
\hline 136 & 1347.2753 & 319.5 & 8.28 \\
\hline 137 & 1347.3391 & 491.7 & 2.12 \\
\hline 138 & 1347.4334 & 396.3 & 9.55 \\
\hline 139 & 1347.5908 & 326.9 & 19.73 \\
\hline 140 & 1348.4951 & 445.8 & 42.47 \\
\hline 141 & 1348.5686 & 479.9 & 7.37 \\
\hline 142 & 1348.5951 & 55.8 & 0.01 \\
\hline 143 & 1349.4535 & 1209.8 & 8.58 \\
\hline 144 & 1349.8225 & 855.3 & 24.96 \\
\hline 145 & 1350.0212 & 2444.4 & 23.85 \\
\hline 146 & 1350.0812 & 1351.2 & 176.66 \\
\hline 147 & 1350.1463 & 5308.9 & 4.98 \\
\hline
\end{tabular}

Table B.1. continued.

\begin{tabular}{|c|c|c|c|}
\hline $\begin{array}{l}\text { Flare } \\
\text { index }\end{array}$ & $\begin{array}{c}t_{\text {peak }} \\
\text { TBJD }\end{array}$ & $\begin{array}{r}\text { Amplitude } \\
\mathrm{e}^{-} \mathrm{s}^{-1} \\
\end{array}$ & $\begin{array}{r}F W H M \\
\min \\
\end{array}$ \\
\hline 148 & 1350.2853 & 1769.8 & 11.17 \\
\hline 149 & 1350.2957 & 2460.0 & 12.99 \\
\hline 150 & 1350.3062 & 588.5 & 10.67 \\
\hline 151 & 1350.3212 & 392.3 & 7.01 \\
\hline 152 & 1350.5975 & 595.6 & 18.42 \\
\hline 153 & 1350.7887 & 347.2 & 4.36 \\
\hline 154 & 1351.7021 & 258.3 & 49.28 \\
\hline 155 & 1352.0038 & 254.4 & 59.49 \\
\hline 156 & 1352.3524 & 1573.3 & 3.12 \\
\hline 157 & 1352.6254 & 191.1 & 31.84 \\
\hline 158 & 1352.7400 & 228.6 & 28.74 \\
\hline 159 & 1352.9386 & 1629.7 & 0.43 \\
\hline 160 & 1352.9528 & 486.5 & 18.27 \\
\hline 161 & 1352.9878 & 437.3 & 49.39 \\
\hline 162 & 1353.0282 & 250.0 & 13.94 \\
\hline 163 & 2036.7060 & 1678.0 & 2.81 \\
\hline 164 & 2036.9803 & 557.7 & 22.15 \\
\hline 165 & 2037.0062 & 731.2 & 8.63 \\
\hline 166 & 2037.0445 & 528.1 & 119.19 \\
\hline 167 & 2037.4443 & 2482.4 & 5.59 \\
\hline 168 & 2037.4926 & 529.0 & 6.16 \\
\hline 169 & 2037.5567 & 629.3 & 0.58 \\
\hline 170 & 2037.6398 & 455.7 & 1.57 \\
\hline 171 & 2037.6661 & 285.1 & 0.07 \\
\hline 172 & 2037.6752 & 11108.5 & 2.09 \\
\hline 173 & 2037.6782 & 1725.8 & 10.30 \\
\hline 174 & 2037.6794 & 2650.0 & 22.55 \\
\hline 175 & 2037.7202 & 4475.7 & 3.33 \\
\hline 176 & 2037.7460 & 313.6 & 9.43 \\
\hline 177 & 2038.1988 & 160.4 & 44.15 \\
\hline 178 & 2039.1836 & 4086.6 & 4.74 \\
\hline 179 & 2039.2663 & 1637.8 & 1.89 \\
\hline 180 & 2039.3501 & 3249.4 & 5.30 \\
\hline 181 & 2039.3868 & 298.5 & 8.35 \\
\hline 182 & 2039.4411 & 733.2 & 10.44 \\
\hline 183 & 2039.5980 & 502.6 & 19.24 \\
\hline 184 & 2039.8267 & 522.3 & 17.25 \\
\hline 185 & 2039.8213 & 1015.2 & 3.54 \\
\hline 186 & 2039.9915 & 538.1 & 176.11 \\
\hline 187 & 2040.0245 & 560.2 & 20.59 \\
\hline 188 & 2040.3473 & 403.2 & 1.96 \\
\hline 189 & 2040.3548 & 313.3 & 2.82 \\
\hline 190 & 2040.7923 & 594.5 & 20.55 \\
\hline 191 & 2041.0997 & 231.1 & 53.67 \\
\hline 192 & 2041.3700 & 871.5 & 3.82 \\
\hline 193 & 2041.6072 & 409.8 & 9.48 \\
\hline 194 & 2041.7500 & 118.8 & 33.72 \\
\hline 195 & 2041.9375 & 333.8 & 45.71 \\
\hline 196 & 2041.9876 & 387.4 & 56.35 \\
\hline 197 & 2042.0502 & 407.3 & 19.53 \\
\hline 198 & 2042.4858 & 5650.2 & 4.36 \\
\hline 199 & 2042.5031 & 3220.7 & 8.76 \\
\hline 200 & 2042.6066 & 532.7 & 8.93 \\
\hline 201 & 2042.6998 & 522.8 & 1.64 \\
\hline 202 & 2043.3819 & 228.0 & 2.52 \\
\hline 203 & 2043.7737 & 266.4 & 48.63 \\
\hline 204 & 2044.0924 & 532.7 & 13.80 \\
\hline 205 & 2044.3488 & 540.4 & 3.00 \\
\hline 206 & 2044.5147 & 371.3 & 30.05 \\
\hline
\end{tabular}


E. Martioli et al.: New constraints on the young planetary system AU Mic

Table B.1. continued.

\begin{tabular}{|c|c|c|c|}
\hline $\begin{array}{l}\text { Flare } \\
\text { index }\end{array}$ & $\begin{array}{c}t_{\text {peak }} \\
\text { TBJD }\end{array}$ & $\begin{array}{r}\text { Amplitude } \\
\mathrm{e}^{-} \mathrm{s}^{-1} \\
\end{array}$ & $\begin{array}{r}F W H M \\
\min \\
\end{array}$ \\
\hline 207 & 2044.5761 & 600.4 & 34.88 \\
\hline 208 & 2044.7252 & 313.4 & 33.65 \\
\hline 209 & 2045.0332 & 163.6 & 8.18 \\
\hline 210 & 2045.3312 & 610.8 & 2.30 \\
\hline 211 & 2045.6075 & 242.5 & 24.26 \\
\hline 212 & 2045.7409 & 408.9 & 3.56 \\
\hline 213 & 2045.8442 & 351.2 & 34.24 \\
\hline 214 & 2046.3703 & 316.6 & 24.52 \\
\hline 215 & 2046.5418 & 284.7 & 95.80 \\
\hline 216 & 2046.6323 & 682.6 & 40.90 \\
\hline 217 & 2046.8676 & 806.2 & 2.98 \\
\hline 218 & 2047.0374 & 191.9 & 6.36 \\
\hline 219 & 2047.0975 & 270.8 & 8.22 \\
\hline 220 & 2047.2069 & 184.4 & 0.04 \\
\hline 221 & 2047.2155 & 3395.1 & 7.50 \\
\hline 222 & 2047.2688 & 340.0 & 38.58 \\
\hline 223 & 2047.3482 & 2740.9 & 9.52 \\
\hline 224 & 2047.4462 & 891.1 & 48.97 \\
\hline 225 & 2047.5051 & 1200.7 & 3.31 \\
\hline 226 & 2047.5481 & 539.1 & 2.23 \\
\hline 227 & 2047.6301 & 1138.6 & 9.53 \\
\hline 228 & 2047.8328 & 1166.7 & 1.96 \\
\hline 229 & 2048.0525 & 374.4 & 7.57 \\
\hline 230 & 2049.2135 & 206.6 & 26.42 \\
\hline 231 & 2049.5213 & 216.6 & 4.62 \\
\hline 232 & 2049.7238 & 206.7 & 3.53 \\
\hline 233 & 2049.7380 & 2365.4 & 2.16 \\
\hline 234 & 2049.7424 & 274.2 & 13.64 \\
\hline 235 & 2049.7546 & 468.5 & 8.35 \\
\hline 236 & 2049.9163 & 84.0 & 182.42 \\
\hline 237 & 2049.7972 & 280.8 & 4.41 \\
\hline 238 & 2050.3662 & 460.2 & 11.07 \\
\hline 239 & 2050.6606 & 453.6 & 18.94 \\
\hline 240 & 2050.7854 & 309.4 & 36.35 \\
\hline 241 & 2050.8613 & 1335.7 & 14.50 \\
\hline 242 & 2050.9074 & 396.0 & 22.97 \\
\hline 243 & 2051.1673 & 928.6 & 12.95 \\
\hline 244 & 2051.1775 & 523.8 & 18.06 \\
\hline 245 & 2051.4456 & 2285.7 & 10.79 \\
\hline 246 & 2051.4575 & 1210.5 & 40.84 \\
\hline 247 & 2051.5155 & 804.9 & 22.33 \\
\hline 248 & 2051.5398 & 491.4 & 66.83 \\
\hline 249 & 2051.8395 & 846.2 & 7.55 \\
\hline 250 & 2052.2513 & 387.8 & 5.20 \\
\hline 251 & 2052.3527 & 337.5 & 18.29 \\
\hline 252 & 2052.6859 & 526.4 & 28.58 \\
\hline 253 & 2052.8846 & 227.8 & 18.12 \\
\hline 254 & 2053.0858 & 588.8 & 2.19 \\
\hline 255 & 2053.2934 & 416.3 & 131.31 \\
\hline 256 & 2053.4293 & 11831.0 & 3.11 \\
\hline 257 & 2053.4313 & 4392.0 & 5.98 \\
\hline 258 & 2053.4364 & 2771.1 & 21.72 \\
\hline 259 & 2053.4471 & 1727.0 & 29.15 \\
\hline 260 & 2053.5172 & 655.4 & 27.49 \\
\hline 261 & 2053.7714 & 642.8 & 3.95 \\
\hline 262 & 2053.8511 & 541.5 & 7.36 \\
\hline 263 & 2054.2890 & 2125.9 & 40.06 \\
\hline 264 & 2054.5200 & 247.5 & 416.71 \\
\hline 265 & 2055.0243 & 356.7 & 25.01 \\
\hline
\end{tabular}

Table B.1. continued.

\begin{tabular}{|c|c|c|c|}
\hline $\begin{array}{l}\text { Flare } \\
\text { index }\end{array}$ & $\begin{array}{c}t_{\text {peak }} \\
\text { TBJD }\end{array}$ & $\begin{array}{r}\text { Amplitude } \\
\mathrm{e}^{-} \mathrm{s}^{-1}\end{array}$ & $\begin{array}{r}F W H M \\
\min \end{array}$ \\
\hline 266 & 2055.1195 & 139.0 & 16.47 \\
\hline 267 & 2055.3076 & 305.4 & 3.46 \\
\hline 268 & 2055.4149 & 370.8 & 10.58 \\
\hline 269 & 2055.5277 & 365.8 & 9.81 \\
\hline 270 & 2055.6639 & 278.9 & 66.80 \\
\hline 271 & 2055.8219 & 257.3 & 34.42 \\
\hline 272 & 2055.9072 & 1427.7 & 23.05 \\
\hline 273 & 2055.9560 & 429.0 & 5.31 \\
\hline 274 & 2056.2248 & 219.2 & 39.11 \\
\hline 275 & 2056.2702 & 4218.7 & 1.21 \\
\hline 276 & 2056.3009 & 648.6 & 22.67 \\
\hline 277 & 2056.3696 & 432.8 & 39.37 \\
\hline 278 & 2056.4690 & 416.0 & 38.42 \\
\hline 279 & 2056.5167 & 515.0 & 15.28 \\
\hline 280 & 2056.6882 & 814.3 & 3.02 \\
\hline 281 & 2056.7114 & 411.5 & 3.34 \\
\hline 282 & 2056.8307 & 3072.5 & 7.83 \\
\hline 283 & 2056.8397 & 1642.1 & 23.47 \\
\hline 284 & 2056.8744 & 556.6 & 35.16 \\
\hline 285 & 2056.9135 & 5101.0 & 4.46 \\
\hline 286 & 2056.9178 & 6950.3 & 21.77 \\
\hline 287 & 2056.9413 & 1903.9 & 72.48 \\
\hline 288 & 2057.0256 & 1115.2 & 165.60 \\
\hline 289 & 2057.1330 & 855.9 & 7.08 \\
\hline 290 & 2057.2076 & 727.6 & 10.93 \\
\hline 291 & 2057.3293 & 335.9 & 1.80 \\
\hline 292 & 2057.6220 & 176.0 & 15.67 \\
\hline 293 & 2057.7999 & 8926.0 & 4.19 \\
\hline 294 & 2057.8206 & 805.8 & 35.86 \\
\hline 295 & 2058.0758 & 474.4 & 18.58 \\
\hline 296 & 2058.1089 & 341.1 & 2.95 \\
\hline 297 & 2058.2392 & 1637.1 & 10.08 \\
\hline 298 & 2058.2417 & 1628.0 & 2.74 \\
\hline 299 & 2058.2491 & 711.4 & 24.72 \\
\hline 300 & 2058.2591 & 642.2 & 20.10 \\
\hline 301 & 2058.2672 & 302.0 & 11.75 \\
\hline 302 & 2058.3522 & 260.7 & 21.24 \\
\hline 303 & 2058.7099 & 937.1 & 2.95 \\
\hline 304 & 2058.7918 & 258.1 & 15.96 \\
\hline 305 & 2058.8039 & 265.8 & 11.94 \\
\hline 306 & 2058.8144 & 272.5 & 15.28 \\
\hline 307 & 2058.8355 & 4984.7 & 0.16 \\
\hline 308 & 2058.8387 & 3972.5 & 7.65 \\
\hline 309 & 2058.8522 & 640.5 & 0.45 \\
\hline 310 & 2058.9145 & 564.9 & 0.32 \\
\hline 311 & 2058.9336 & 276.6 & 2.32 \\
\hline 312 & 2058.9386 & 365.7 & 1.12 \\
\hline 313 & 2059.0986 & 473.3 & 1.64 \\
\hline 314 & 2059.1116 & 628.6 & 58.56 \\
\hline 315 & 2059.3548 & 477.5 & 9.92 \\
\hline 316 & 2059.3865 & 520.4 & 9.98 \\
\hline 317 & 2059.4226 & 274.5 & 4.58 \\
\hline 318 & 2059.5478 & 4219.3 & 1.21 \\
\hline 319 & 2059.5512 & 3627.0 & 6.71 \\
\hline 320 & 2059.6466 & 351.4 & 16.89 \\
\hline 321 & 2059.6786 & 504.1 & 11.39 \\
\hline 322 & 2060.2895 & 493.0 & 1.21 \\
\hline 323 & 2060.4086 & 913.3 & 2.06 \\
\hline 324 & 2060.5060 & 851.8 & 24.80 \\
\hline
\end{tabular}

A177, page 15 of 19 


\section{Appendix C: MCMC samples and posterior distributions}

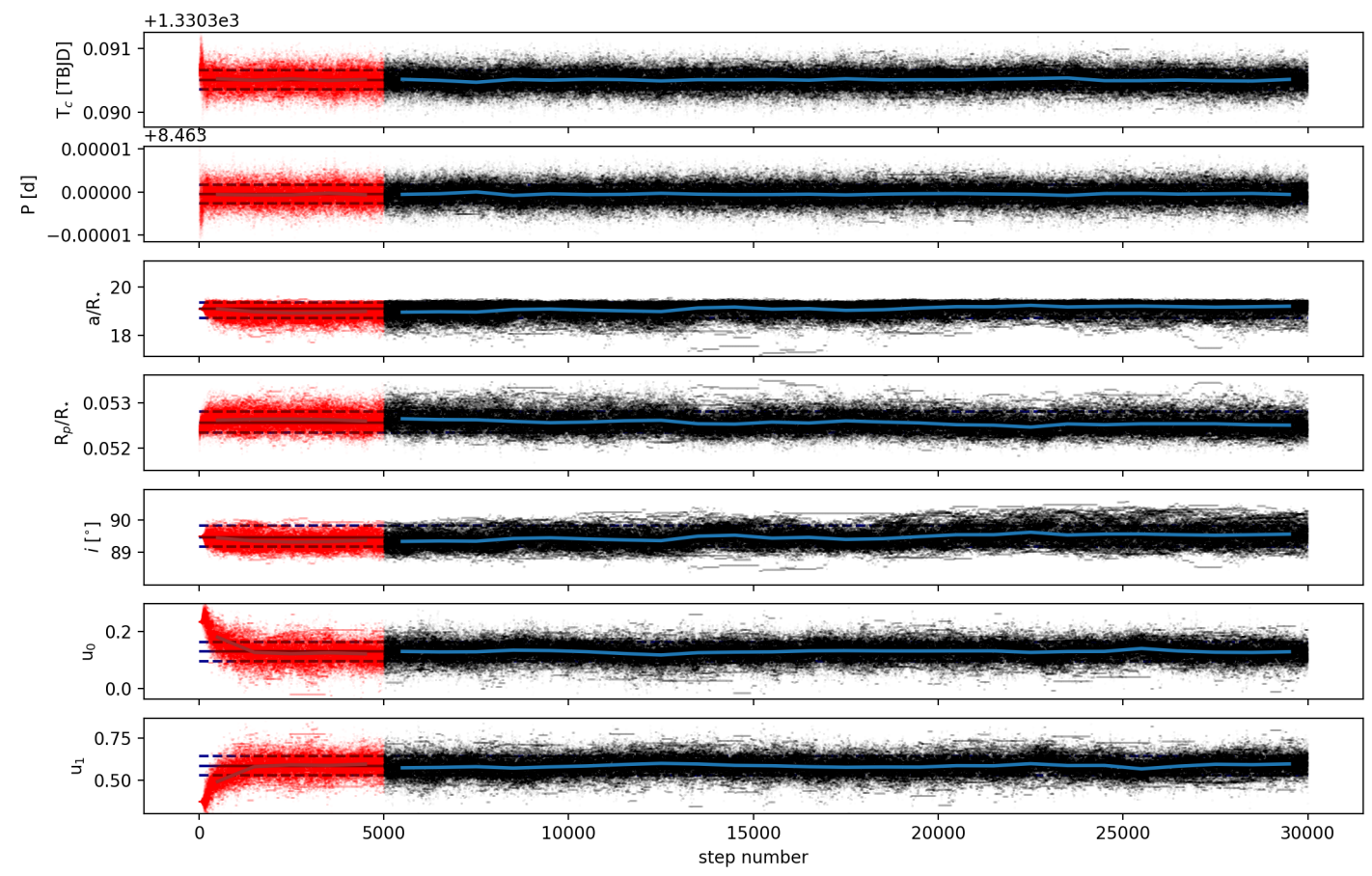

Fig. C.1. MCMC samples (black dots) used to calculate the posterior distributions of the transit parameters of AU Mic b. The red dots show the discarded samples corresponding to the first 5000 steps of the chain. The blue lines show the median calculated for bins of 1000 steps. Dashed lines show the 16- and 84-percentiles and the solid horizontal lines shows the medians of the valid samples.)
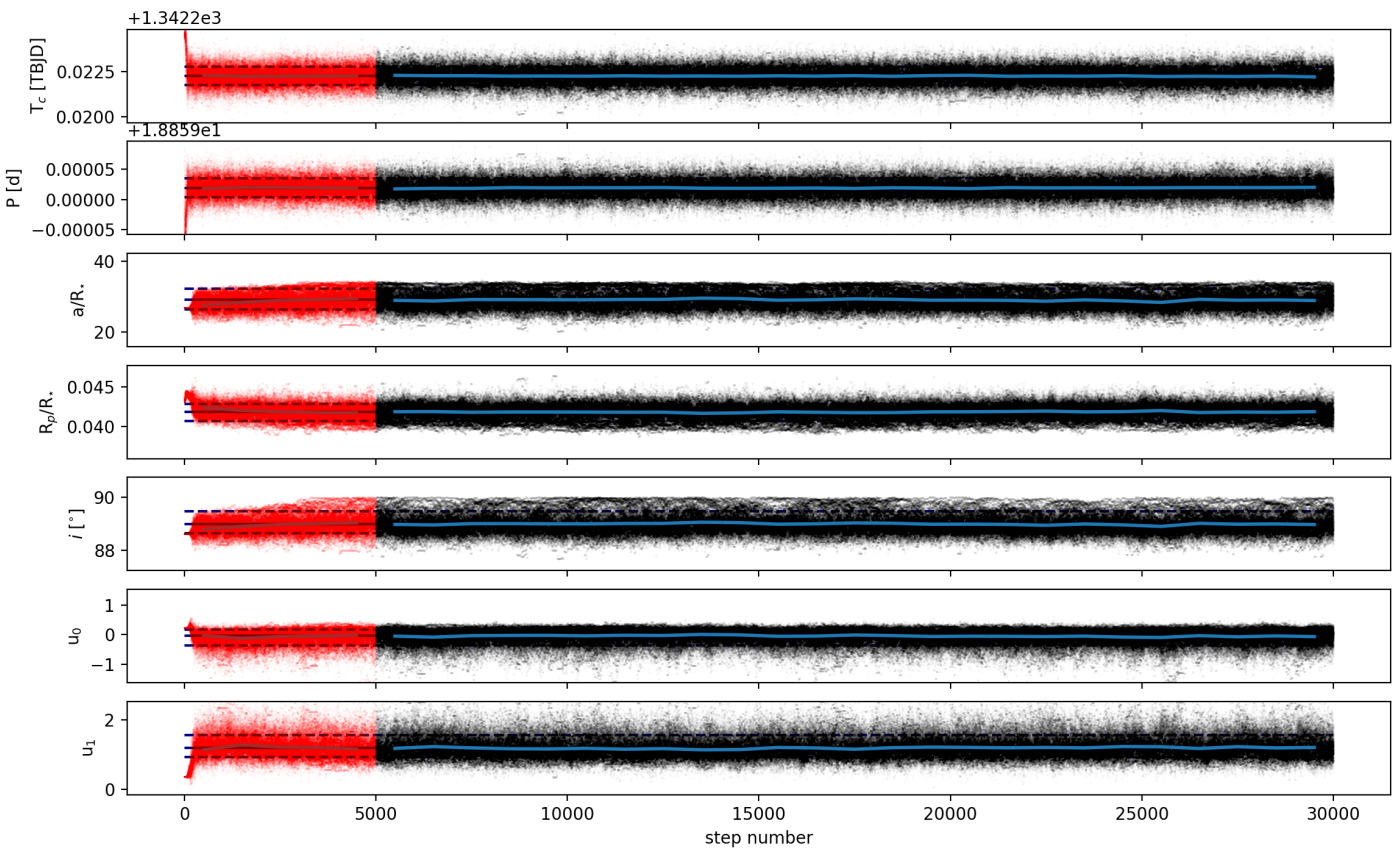

Fig. C.2. Same Fig. C.1 for the MCMC samples of the transit parameters of AU Mic c. 


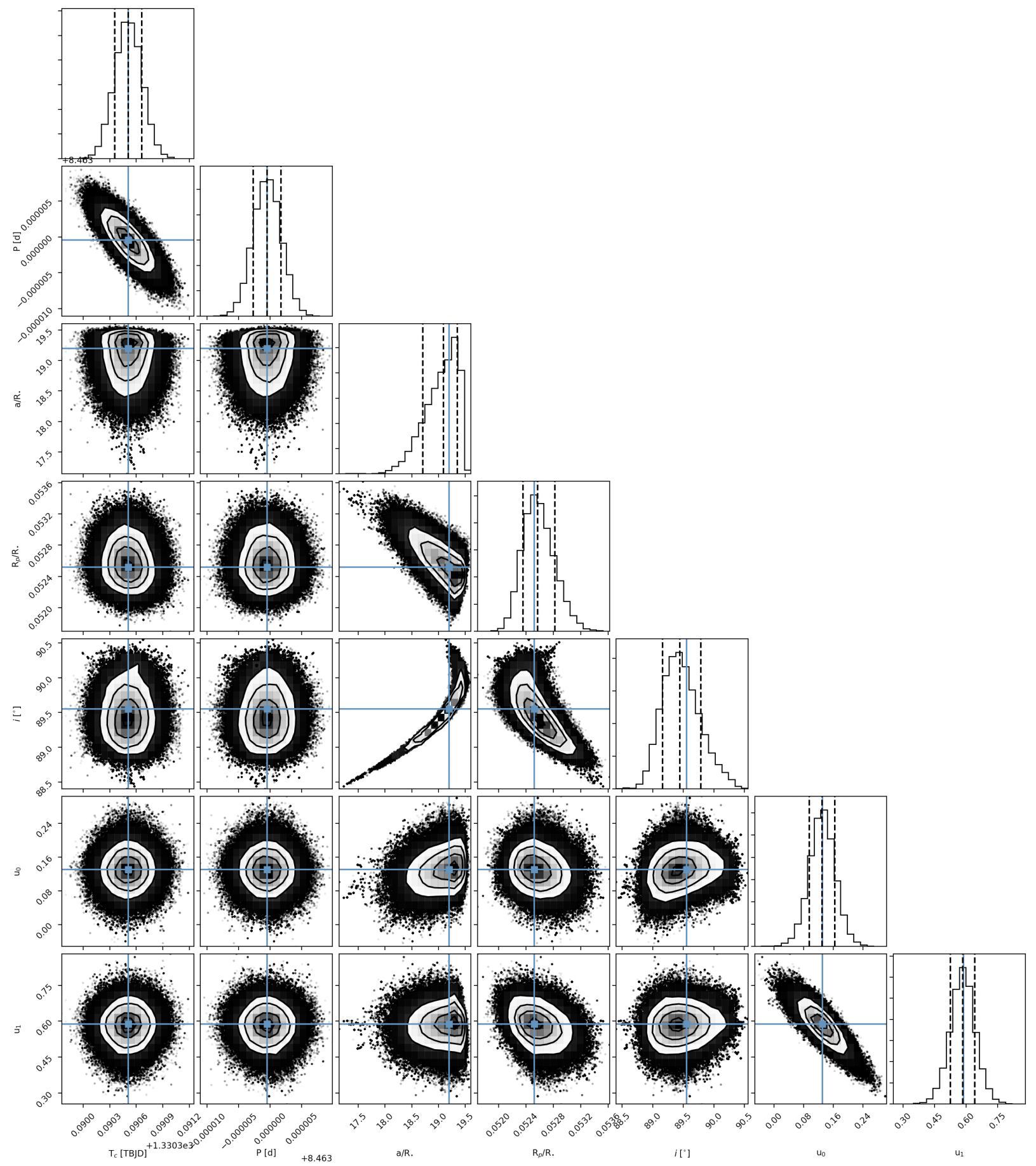

Fig. C.3. Pairs plot showing the MCMC samples and posterior distributions for the transit parameters of AU Mic b as presented in Table 2. The contours mark the 1,2 , and $3 \sigma$ regions of the distribution. The blue crosses indicate the best fit values for each parameter and the dashed vertical lines in the projected distributions show the median values and the $1 \sigma$ uncertainty ( $34 \%$ on each side of the median). 

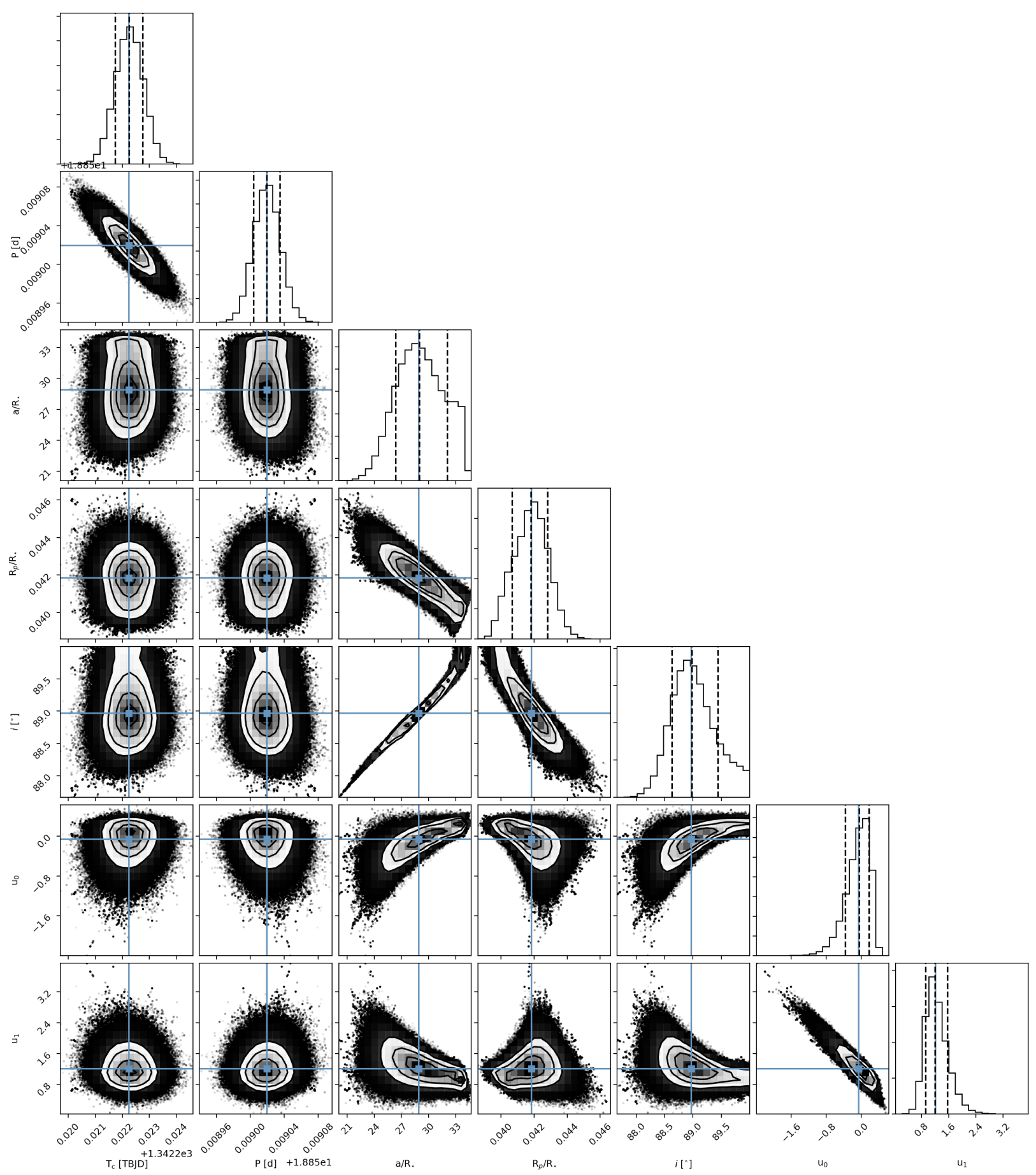

Fig. C.4. Pairs plot showing the MCMC samples and posterior distributions for the transit parameters of AU Mic b as presented in Table 2. Details are the same as in Fig. C.3 for the analysis of the three transits of AU Mic c. 


\section{Appendix D: Independent fit to the transits of AU Mic c}

This appendix presents the results obtained from an MCMC analysis (as in Sect. 5) performed on each transit of AU Mic c independently. Table D.1 shows the posterior of the transit parameters, where we present first the results using broad priors and then using the more restricted priors, as described in Sect. 6. We notice that the period and semi-major axis are not well constrained by the shape of the transits. As a consequence, when using broad priors, their posteriors turn out to have relatively large uncertainties. However, the posterior of all parameters agree within $3 \sigma$ among the three events and they also agree with our derived period of $P \sim 18.86-\mathrm{d}$. The fit parameters for the more constrained priors also agree within $3 \sigma$, and the dispersion of residuals are improved with respect to the unconstrained solution, which supports our hypothesis that these events are caused by the transits of the same planet, namely, AU Mic c.

Table D.1. Fit transit parameters for an independent analysis of each of the three transit-like events identified as AU Mic c in the TESS light curve.

\begin{tabular}{ccccccc}
\hline \hline Event & $T_{0}[\mathrm{TBJD}]$ & $P[\mathrm{~d}]$ & $a / R_{\star}$ & $R_{\mathrm{p}} / R_{\star}$ & $i_{\mathrm{p}}\left[^{\circ}\right]$ & $\sigma[\mathrm{ppm}]$ \\
\hline $1^{(a)}$ & $1342.22 \pm 0.03$ & $30 \pm 6$ & $40 \pm 8$ & $0.028 \pm 0.006$ & $89.28 \pm 0.45$ & - \\
\hline 1 & $1342.2247_{-0.0004}^{+0.0005}$ & $17_{-8}^{+13}$ & $25_{-11}^{+18}$ & $0.036_{-0.0012}^{+0.0014}$ & $88.6_{-1.2}^{+0.9}$ & 388 \\
2 & $2040.0063_{-0.0004}^{+0.0005}$ & $23_{-5}^{+7}$ & $27 \pm 6$ & $0.0420_{-0.0009}^{+0.0007}$ & $88.28_{-0.5}^{+0.3}$ & 678 \\
3 & $2058.8672 \pm 0.0004$ & $21_{-12}^{+46}$ & $37_{-21}^{+78}$ & $0.0359_{-0.0005}^{+0.0009}$ & $89.35_{-1.03}^{+0.52}$ & 646 \\
\hline 1 & $1342.2243_{-0.0006}^{+0.0004}$ & $18.8574_{-0.0008}^{+0.0016}$ & $31.3_{-0.8}^{+0.4}$ & $0.0393_{-0.0004}^{+0.0005}$ & $89.04_{-0.06}^{+0.09}$ & 355 \\
2 & $2040.0062 \pm 0.0003$ & $18.8588_{-0.00014}^{+0.00012}$ & $23.8_{-1.3}^{+1.5}$ & $0.0444 \pm 0.0007$ & $88.2 \pm 0.2$ & 650 \\
3 & $2058.8671 \pm 0.0004$ & $18.8592_{-0.0003}^{+0.0002}$ & $31.5_{-2.1}^{+0.2}$ & $0.0409 \pm 0.0004$ & $89.03_{-0.20}^{+0.07}$ & 610 \\
\hline
\end{tabular}

Notes. The first row shows the previous results for Event 1 by Plavchan et al. (2020), the three following rows show the best fit parameters from our MCMC analysis of each event adopting an orbital period with an uniform prior of $P=\mathcal{U}(1,500) \mathrm{d}$. The last three rows show the fit parameters using an orbital period with a normal prior of $P=\mathcal{N}(18.85895,0.00003) \mathrm{d}$, as described in the text. ${ }^{(a)}$ Fit parameters for Event 1 obtained by Plavchan et al. (2020). 